\title{
HI observations of nearby galaxies
}

\section{The first list of the Karachentsev catalog}

\author{
W.K. Huchtmeier ${ }^{1}$, I.D. Karachentsev ${ }^{2}$, V.E. Karachentseva ${ }^{3}$, and M. Ehle ${ }^{4}$ \\ 1 Max-Planck-Institut für Radioastronomie, Auf dem Hügel 69, D-53121 Bonn, Germany \\ 2 Special Astrophysical Observatory, Russian Academy of Sciences, N. Arkhyz, KChR, 357147, Russia \\ 3 Astronomical Observatory of Kiev University, Kiev, Ukraine \\ 4 Max-Planck-Institut für Extraterrestrische Physik, Giessenbachstraße, D-85740 Garching bei München, Germany
}

Received September 14; accepted November 16, 1999

\begin{abstract}
We present HI observations of the galaxies in the first list of the Karachentsev catalog of previously unknown nearby dwarf galaxies (Karachentseva \& Karachentsev 1998). This survey covers all known nearby galaxy groups within the Local Volume (i.e. within $10 \mathrm{Mpc}$ ) and their environment, that is about $25 \%$ of the total sky. A total of 257 galaxies have been observed with a detection rate of $60 \%$. We searched a frequency band corresponding to heliocentric radial velocities from $-470 \mathrm{kms}^{-1}$ to $\sim+4000 \mathrm{~km} \mathrm{~s}^{-1}$. Non-detections are either due to limited coverage in radial velocity, confusion with Local HI (mainly in the velocity range $-140 \mathrm{~km} \mathrm{~s}^{-1}$ to $+20 \mathrm{~km} \mathrm{~s}^{-1}$ ), or lack of sensitivity for very weak emission. $25 \%$ of the detected galaxies are located within the Local Volume. Those galaxies are dwarf galaxies judged by their optical linear diameter $(1.4 \pm 0.2 \mathrm{kpc}$ on the average), their mean total $\mathrm{HI}$ mass $\left(4.610^{7} M_{\odot}\right)$, and their observed linewidths $\left(39 \mathrm{~km} \mathrm{~s}^{-1}\right)$.
\end{abstract}

Key words: galaxies: distances and redshifts; dwarf; fundamental parameters; general

\section{Introduction}

The only way to study the smallest galaxies is to search for them in our cosmic neighborhood. The first systematic catalog of nearby galaxies was prepared by Kraan-Korteweg \& Tammann (1979) who collected all known galaxies with corrected radial velocities $v_{0}$ $\leq 500 \mathrm{~km} \mathrm{~s}^{-1}$, a total of 179 objects (hereafter called the KKT sample). Since that time the number of known galaxies within the Local Volume (i.e. within a distance

Send offprint requests to: W.K. Huchtmeier,

e-mail: huchtmeier@mpifr-bonn.mpg.de of $10 \mathrm{Mpc}$ ) increased to 303 objects (Karachentsev et al. 1999). For the past decade the initial KKT sample has been increased almost two times in number due to the mass redshift surveys of galaxies from the known catalogues, revealing new nearby galaxies in the Milky Way "Zone of Avoidance", as well as special searches for dwarf galaxies in nearby groups. The increasing numbers of galaxies in the Local Volume is mainly due to many new dwarf galaxies. This fact demonstrates how incomplete our knowledge about the galaxy population of even the Local Volume is.

A couple of years ago Karachentseva \& Karachentsev (1998; hereafter KK98) initiated an all-sky search for candidates for new nearby dwarf galaxies using the second Palomar Sky Survey and the ESO/SERC plates of the southern sky. The results of the first two segments of the survey have been published, they cover large areas around the known galaxy groups in the Local Volume (KK98) and the area of the Local Void (Karachentseva et al. 1999). In a next step to derive distances we will measure radial velocities. Later on we will aim for more exact photometric distances. In this paper we present the first follow-up observations, the HI search for the galaxies in KK98. The HI search for dwarf irregular galaxies seems quite efficient as these galaxies are HI rich in general and with adequate velocity resolution, say $5 \mathrm{~km} \mathrm{~s}^{-1}$, all the $\mathrm{HI}$ of a given galaxy will be within a few velocity channels. The characteristic signature of a dwarf galaxy profile, a nearly gaussian structure, is different from radio interference and easily will lead to a good signal-to-noise ratio.

\section{Observations}

Observations were performed with three different radio telescopes for different declination ranges. The $100-\mathrm{m}$ radiotelescope at Effelsberg was used for declinations greater 

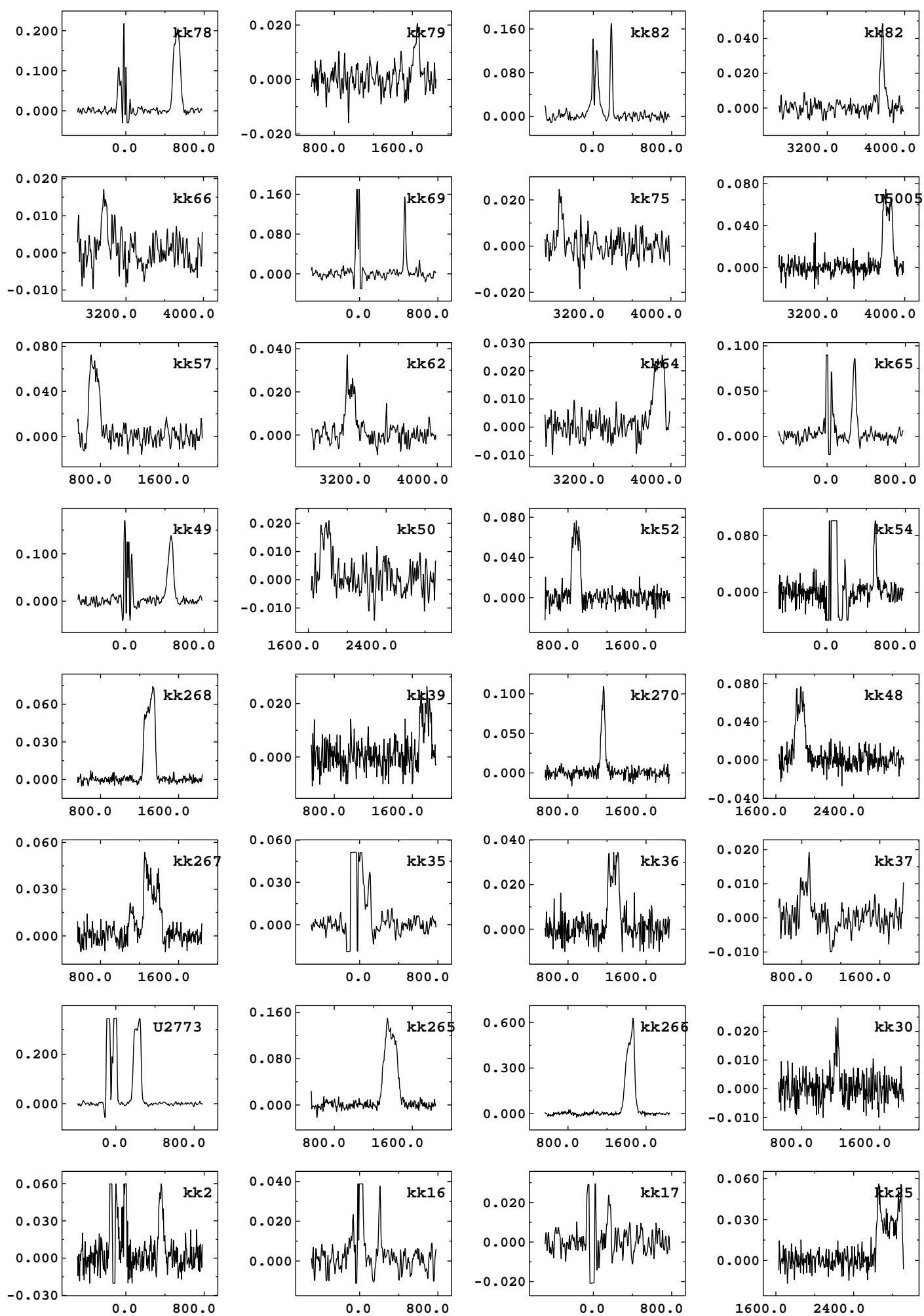

Fig. 1. HI profiles observed with the 100-m radio telescope at Effelsberg which has a HPBW of 9.3 at a wavelength of $21 \mathrm{~cm}$. Observations were obtained in the total power mode [ON - OFF] which yields a residual of the Local HI emission around $0 \mathrm{~km} \mathrm{~s}^{-1}$. The profiles are arranged in ascending R.A. starting at the bottom left corner 

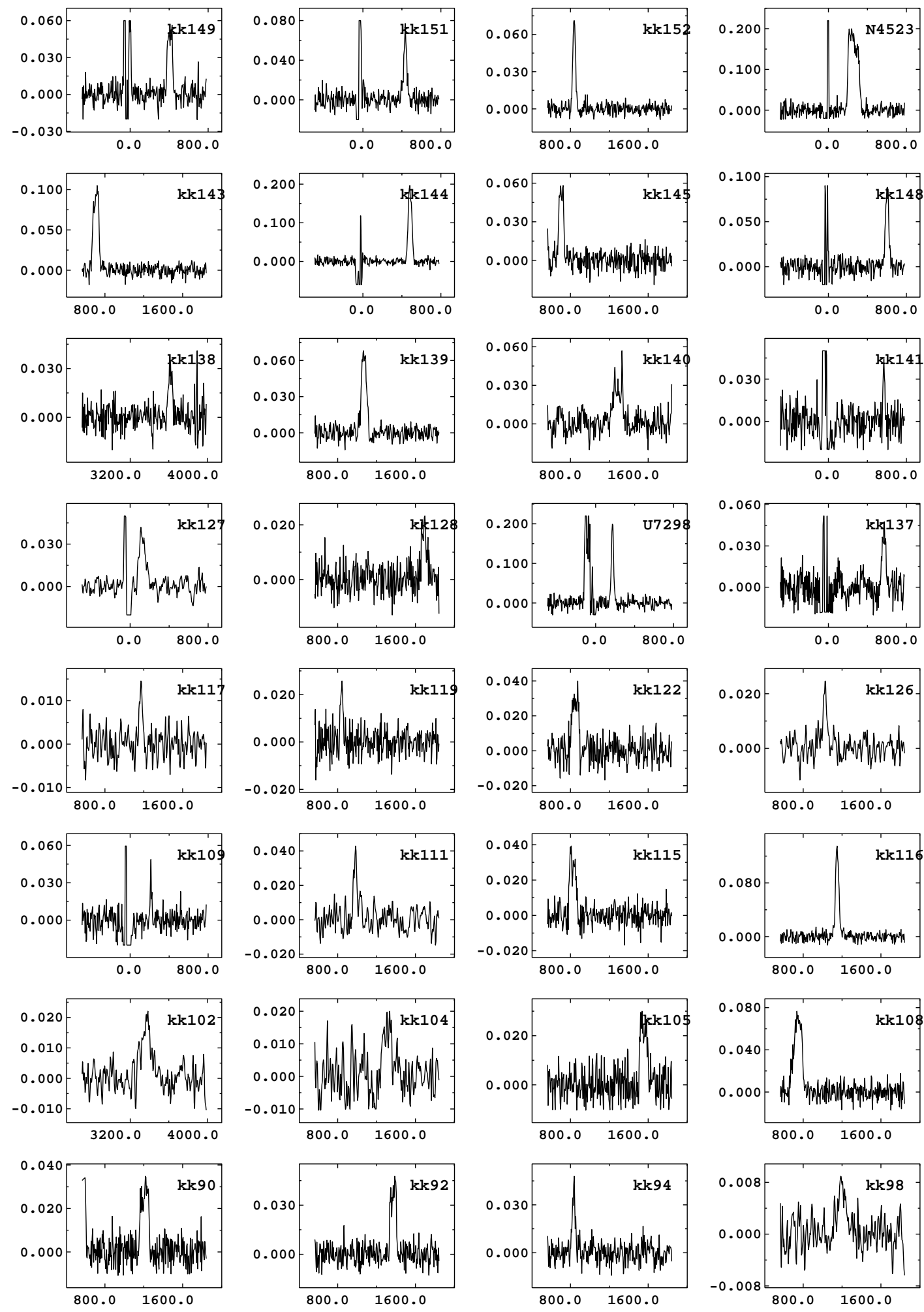

Fig. 1. continued 

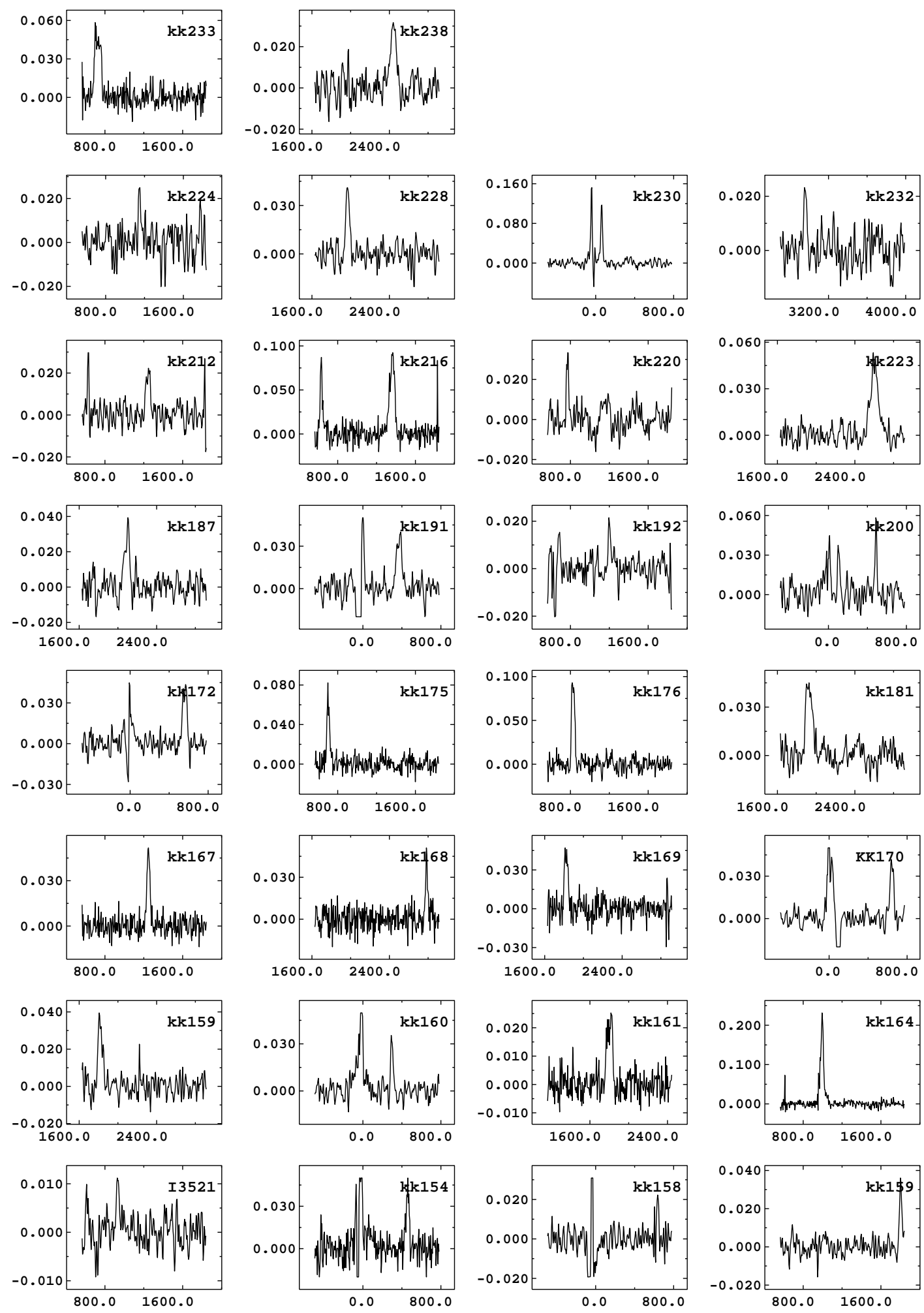

Fig. 1. continued 

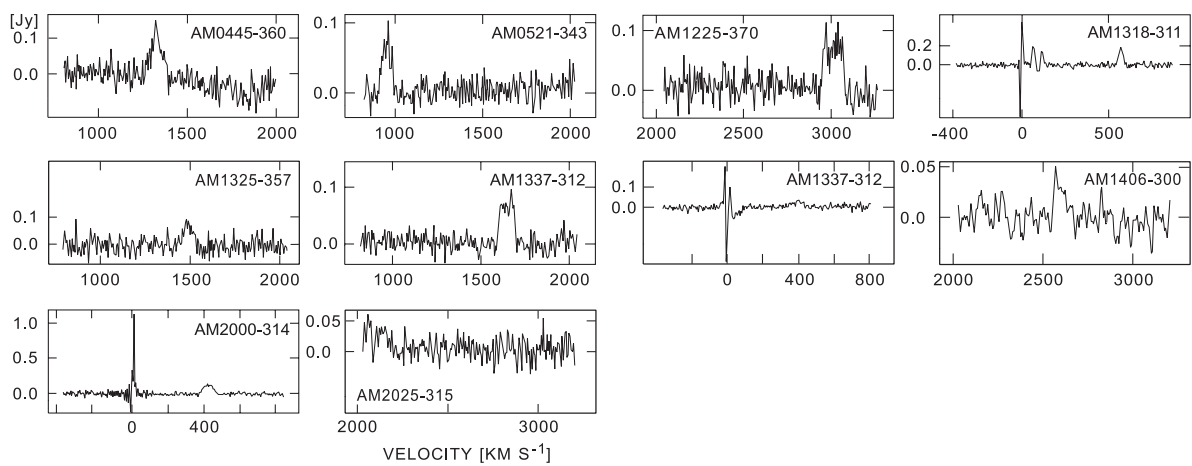

Fig. 2. HI profiles observed with the Nançay radio telescope (HPBW of $3.6 \times 22^{\prime}$ for the declination range in question)

than $-31^{\circ}$, the Nançay radio telescope was selected for galaxies in the declination range $-38^{\circ} \leq-31^{\circ}$, and the compact array of the Australia Telescope was used for galaxies south of $-38^{\circ}$.

\subsection{Effelsberg observations}

The radio telescope at Effelsberg has been used in the total power mode $(\mathrm{ON}-\mathrm{OFF}$ ) combining a reference field 5 min earlier in R.A. with the on-source position. A dual channel HEMT receiver had a system noise of $30 \mathrm{~K}$.

The 1024 channel autocorrelator was split into 4 bands (bandwidth $6.25 \mathrm{MHz}$ ) of 256 channels each shifted in frequency by $5 \mathrm{MHz}$ with respect to their neighbor in order to cover a velocity range from -470 to $3970 \mathrm{~km} \mathrm{~s}^{-1}$ overlapping $1.5 \mathrm{MHz}$ between channels. The resulting channel separation was $5.1 \mathrm{~km} \mathrm{~s}^{-1}$ yielding a resolution of $6.2 \mathrm{~km} \mathrm{~s}^{-1}$ (10.2 $\mathrm{km} \mathrm{s}^{-1}$ after Hanning smoothing). The HI profiles observed with the $100-\mathrm{m}$ radiotelescope are presented in Fig. 1 in order of increasing R.A. as in Table 1. The half power beam widths (HPBW) of the Effelsberg telescope at this wavelength is 9 !'3.

\subsection{Nançay observations}

For 15 galaxies in the declination range $-38^{\circ} \leq-31^{\circ}$ the Nançay radio telescope was used with the same velocity resolution and coverage. Major differences to the description given for the Effelsberg observations were a different system noise $(45 \mathrm{~K})$, a different antenna beam $\left(3 ! 6 \times 22^{\prime}\right.$ in R.A. and Dec. for this declination range), and shorter integration phases with a cycle of 2 minutes for the $\mathrm{ON}$ and the OFF positions. Nine galaxies have been detected (Fig. 2).

\subsection{Compact Array of the Australia Telescope}

40 of the 57 galaxies south of declination $-38^{\circ}$ have been observed with the Compact Array of the Australia
Telescope. For this HI search we have chosen the $750 \mathrm{~A}$ antenna array configuration in order to yield an antenna beam comparable to the optical size of the smallest galaxies (i.e. $\sim 1^{\prime}$ ). The frequency setup and correlator configuration was such that we obtained a velocity coverage from -450 to $+2900 \mathrm{~km} \mathrm{~s}^{-1}$ and a channel separation of $6.6 \mathrm{~km} \mathrm{~s}^{-1}$ (i.e. a resolution of $7.9 \mathrm{~km} \mathrm{~s}^{-1}$ ). Each galaxy was observed for 10 min every few hours. With five to six observations per target position we achieved a regular coverage of the $u v$ plane for these "snapshot mode" observations. The resulting integrated HI profiles are given in Fig. 3 (for a more detailed discussion of these data see Huchtmeier et al. in preparation). We may miss some flux with the interferometer (missing flux) as the observed HI emission extends over more than $2^{\prime}$ per channel for over $60 \%$ of the galaxies. Galaxies from the kk98 sample not observed are: kk 11, kk 63, kk179, kk 184, kk 189, kk 190，kk197， kk203，kk211，kk213，kk214, kk 217, kk 221, kk 222, kk 235, kk 244, kk 248.

\section{The data}

Our search list was an early version of the list of KK98 containing a few additional galaxies which did not make it into the final version because of their morphology and/or size (i.e. they were too small). Particularly, we took into account the results of $\mathrm{HI}$ searches for nearby dwarf galaxies made by Kraan-Korteweg et al. (1994), Huchtmeier et al. (1995), Burton et al. (1996), Huchtmeier \& van Driel (1996), Huchtmeier et al. (1997) and Cote et al. (1997). The optical data of our galaxies are given in Table 1 . The kk-number (or other identification if there is no kknumber) is given in Col. 1, R.A. and Dec. (1950) follow in Cols. 2 and 3. The optical diameters $a$ and $b$ in the de Vaucouleurs $\left(D_{25}\right)$ system follow in Cols. 4 and 5, the morphological type in Col. 6 where we use the following coding:

Im - irregular blue object with bright knot(s);

Ir - irregular without knots or with amorphous condensations, the colour is neutral or bluish; 

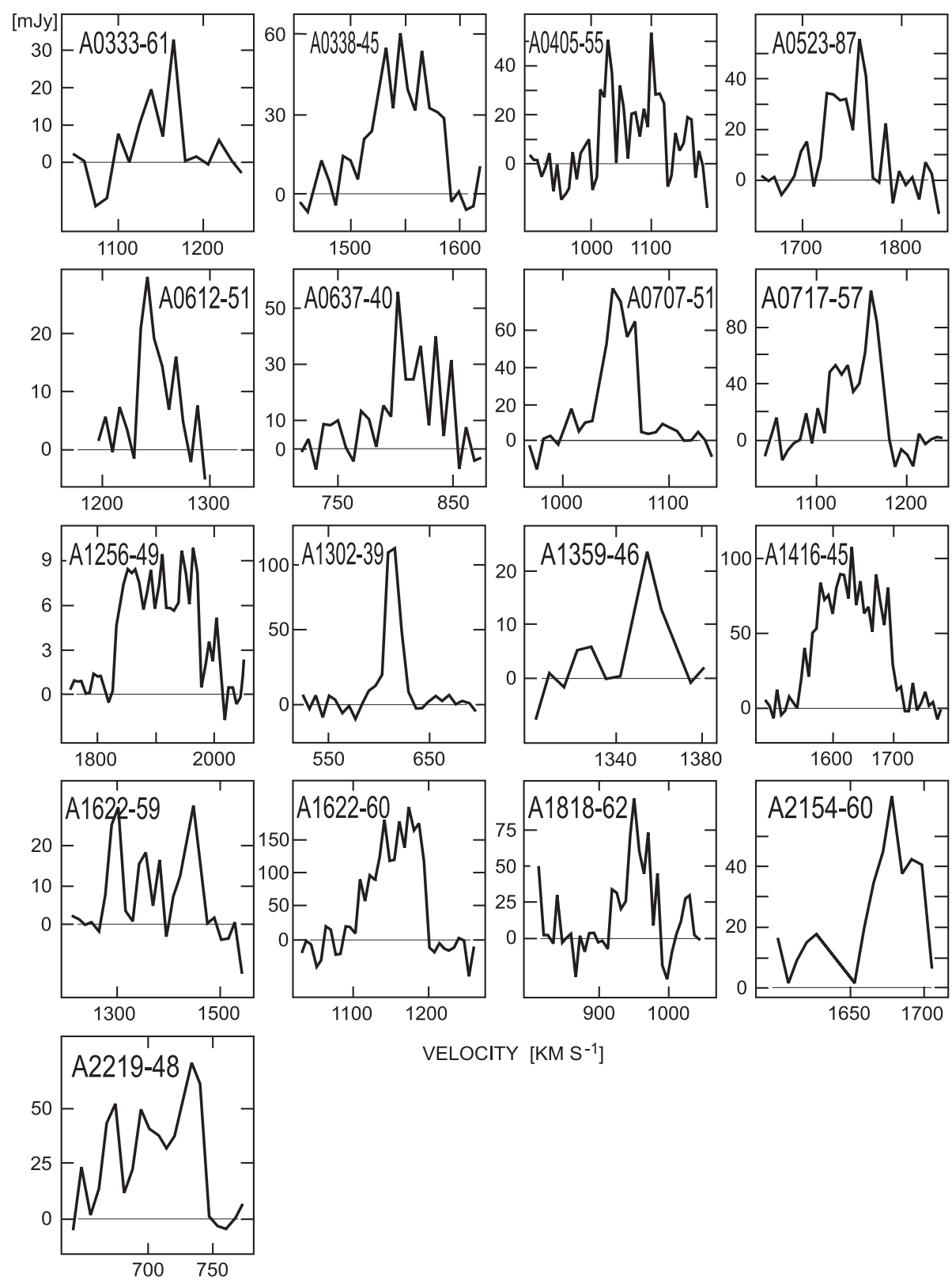

VELOCITY $\left[\mathrm{KM} \mathrm{S}^{-1}\right]$

Fig. 3. HI profiles observed with the Australia Telescope Compact Array. The synthesized antenna beam is of the order of $1^{\prime}$

Sm - disturbed spiral or irregular with signs of spiral structure;

Sph - spheroidal, with very low brightness gradient or without any, the color is neutral or redish.

The optical surface brightness (SB) has been coded (see KK98): high (H), low (L), very low (VL), and extremely low (EL) in Col. 7. The total blue magnitude $B_{\mathrm{t}}$ and its reference follow in Cols. 8 and 9. "NED" - data are from the NASA/Extragalactic Database, "IK" - visual estimates from POSS (typical error is about $0.4 \mathrm{mag}$ ) by I. Karachentsev, "6 m" - accurate photometric data from the 6 - $\mathrm{m}$ telescope CCD-frames obtained by Karachentsev and coworkers (unpublished); "UH" - photometric data from U. Hopp (Calar Alto) unpublished. The Galactic extinction follows in Col. 10. Other names (identifications) are listed in Col. 11.

Results of the HI observations are summarized in Table 2. The kk-number is given in Col. 1, the HI-flux [ $\left.\mathrm{Jy} \mathrm{km} \mathrm{s}^{-1}\right]$ follows in Col. 2, the maximum emission and/or the rms noise [mJy] in Col. 3, the heliocentric radial velocity plus error in Col. 4 , the line widths at the $50 \%$, the $25 \%$, and the $20 \%$ level of the peak emission in Col. 5. Distances (Col. 6) have been derived with different methods, there are photometric distances in 


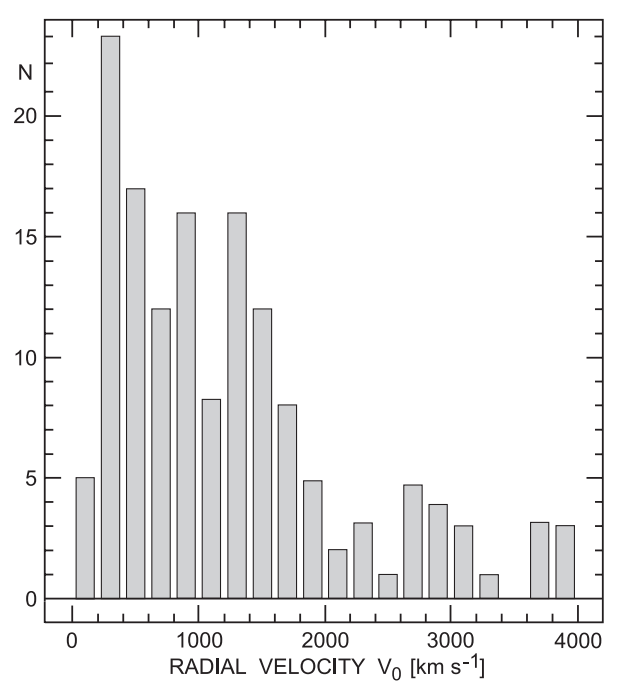

Fig. 4. The histogram shows the number of galaxies per velocity interval of $200 \mathrm{~km} \mathrm{~s}^{-1}$. The distribution of corrected radial velocities $\left(v_{0}\right)$ of our galaxy sample demonstrates the local character of these galaxies

some cases, in other cases the group membership yields a distance. If no other distance estimate is available, we assumed a Hubble constant of $75 \mathrm{~km} \mathrm{~s}^{-1} \mathrm{Mpc}^{-1}$ to derive a "kinematic" distance. The absolute magnitude is given in Col. 7, the integrated HI mass (Col. 8) was calculated as (e.g. Roberts 1969)

$$
\left(M_{\mathrm{HI}} / M_{\odot}\right)=2.35510^{5} \times D^{2} \times \int S_{v} \mathrm{~d} v
$$

where $D$ is the distance of the galaxy in Mpc and $\int S_{v} \mathrm{~d} v$ is the integrated HI flux in $\mathrm{Jy} \mathrm{km} \mathrm{s}^{-1}$. The relative HI content $M_{\mathrm{HI}} / L_{B}$ follows in Col. 9. Finally, Col. 10 contains comments relative to the telescope used for the observation: unless otherwise noted observations have been performed with the 100-m radiotelescope at Effelsberg, N marks the Nançay radio telescope, ATCA - the Australia Telescope Compact Array at Culgoora, NSW.

In a number of cases emission at negative radial velocities has been observed (kk 20, kk 236, kk 237; only kk 236 has been plotted as an example). The Dwingeloo HI survey (Hartmann \& Burton 1997) has been consulted: in all cases of negative radial velocities extended HI emission was found suggesting that we observed high velocity clouds in our Galaxy.

\section{Discussion}

A great majority (73\%) of our galaxies are of type Im (26) and $\operatorname{Ir}(162)$, about $20 \%$ are of type Sph/Ir (12) and Sph (39), while the rest of $8 \%$ is a collection of different types from spiral to $\mathrm{Im} / \mathrm{Sm}$ and BCD. The detection rate of our sample galaxies depends on the morphological type.

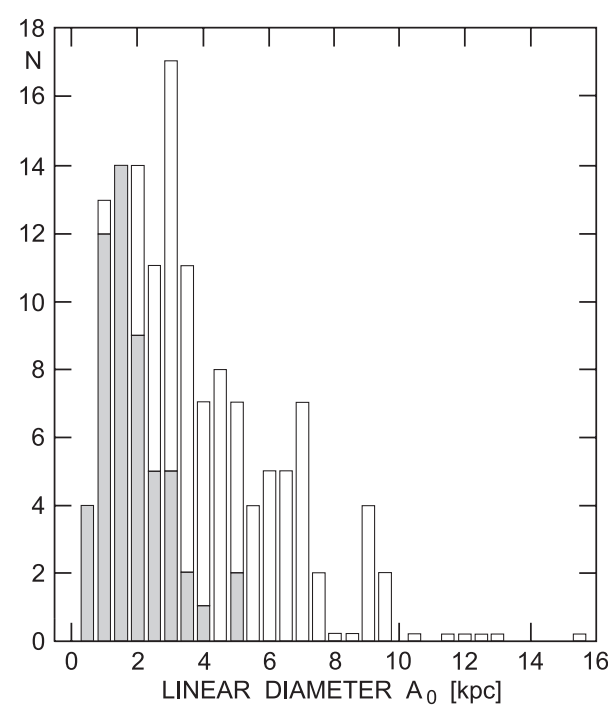

Fig. 5. The distribution of the optical linear diameter $A_{0}$ in $\mathrm{kpc}$ for the whole sample in the de Vaucouleurs $\left(D_{25}\right)$ system is given here. Galaxies within $10 \mathrm{Mpc}$ (i.e. within the Local Volume) are shown by shaded areas. The medium value for the shaded areas is $1.4 \pm 0.2 \mathrm{kpc}$

$75 \%$ of the spirals (type S0 to $\mathrm{Sm} / \mathrm{Im}$ and BCD) were detected; the detection rate for types Im and Ir is very similar close to $60 \%$, whereas the detection rate for types $\mathrm{Sph} / \mathrm{Ir}$ and $\mathrm{Sph}$ is considerable lower at 33 and $23 \%$, respectively. The detection rate depends on the optical surface brightness (SB) class, too. From high SB to low, very low, and extremely low SB the detection rate decreases from $70 \%$ to $58 \%, 49 \%$, and $43 \%$, respectively. This trend reflects the type dependence and the fact that we deal with fainter galaxies as we descend from high SB to very low SB, the median absolute magnitudes for the detected galaxies change from $-15.43(\mathrm{H})$ to -13.92 (VL) for our brightness classes.

A number of the galaxies within the present sample are associated with nearby groups of galaxies (e.g. Tully 1988) according to their position, radial velocity and relative resolution:

NGC 672 group: kk 13, kk 14, kk 15;

NGC 784 group: kk 16, kk 17;

Maffei group: kk 19, kk21, kk 22, kk 23, kk35 kk44;

Orion group: kk 49;

M 81 group: kk 81, kk 83, kk 85, kk 89, kk 89, kk 91;

Leo group: kk 94;

CVn cloud: kk 109, UGC 7298, kk 137, kk 141, kk 144, kk148, kk149, kk 151, kk 154, kk 158, kk 160, kk191, kk 206, kk 220, kk 230;

Centaurus group: kk170, kk179, kk182, kk190, kk 191, kk 195, kk 197, kk 200, kk 211, kk 217, kk 218; NGC 6946 group: kk 250, kk 251, kk 252; 


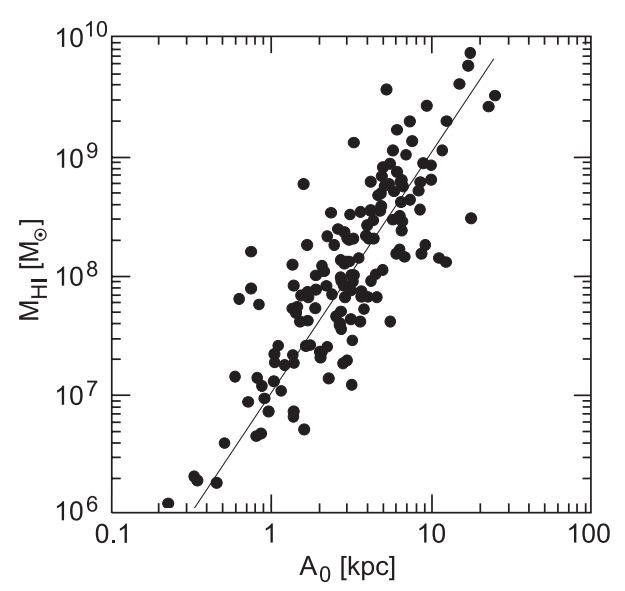

Fig. 6. The total mass of neutral hydrogen $M_{\mathrm{HI}}$ of the galaxies in our sample is plotted versus the linear extent (in $\mathrm{kpc}$ ). The full line represents the regression line for the KKT sample (Huchtmeier \& Richter 1988)

Virgo cluster: kk111, kk127, kk128, kk140, NGC 4523, IC3517, kk164, kk168, kk169, kk172, kk 173, U 8091.

There are a few cases of high $M_{\mathrm{HI}} / L_{B}$ values in Table 2 . Four of the five galaxies with $M_{\mathrm{HI}} / L_{B} \geq 5$ are actually found to be confused by emission from nearby galaxies (see footnotes to Table 2 ).

The present sample of galaxies as presented in Tables 1 and 2 will be discussed now in some detail with the help of global parameters. The distribution of radial velocity $\left(v_{0}\right.$, corrected for the rotation of our galaxy) is given in Fig. 4. Apart from a few background objects most of the galaxies belong to the local supercluster, about $25 \%$ are within the Local Volume. From this situation it is clear that the great majority of the galaxies in the present sample are dwarfish in nature. This will be shown more convincingly below when we compare several other global parameters of these objects.

Next we will look at the optical linear diameter $A_{0}$ (in kpc). The histogram in Fig. 5 presents the number of galaxies binned in intervals of $0.5 \mathrm{kpc}$ width. The distribution of the optical linear diameters of our galaxies extends from $0.2 \mathrm{kpc}$ to $26 \mathrm{kpc}$, yet the great majority is smaller than $8 \mathrm{kpc}$ in diameter (in the de Vaucouleurs $D_{25}$ system). Galaxies in the Local Volume (indicated by shaded areas) are even smaller with a median value of 1.4 $\pm 0.2 \mathrm{kpc}$.

Now we will use the correlation of two global parameters to compare the present sample of galaxies with the previously known galaxies in the Local Volume. In Fig. 6 the total mass of neutral hydrogen $M_{\mathrm{HI}}$ of the galaxies is plotted versus their linear extent $A_{0}$ for this sample of galaxies. The full line is the regression line for the KKT sample (Huchtmeier \& Richter 1988). This regression line seems to be an excellent fit for the present sample, too.
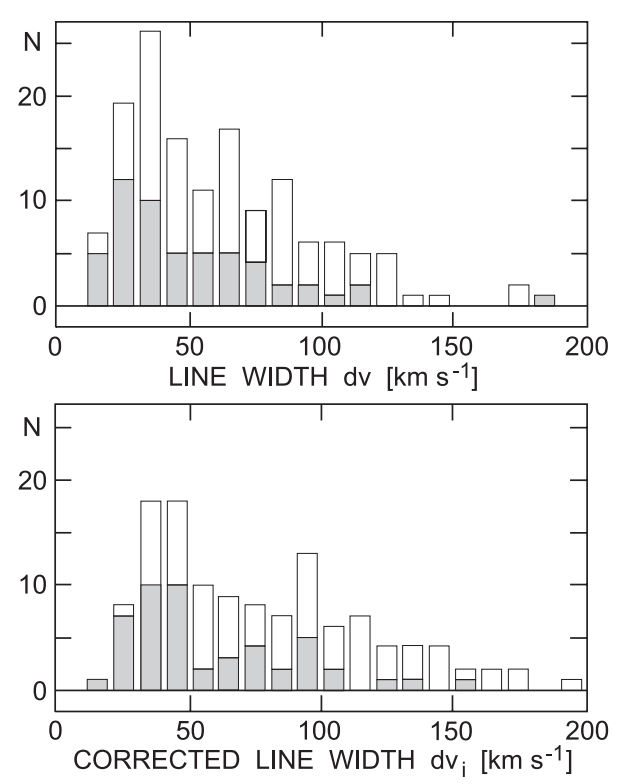

Fig. 7. The distribution of line widths of our galaxy sample is given for the observed values $(\mathrm{d} v)$ in the upper panel and for the (for inclination corrected values $\left(\mathrm{d} v_{i}\right)$ in the lower panel. Galaxies within the Local Volume (i.e. within $10 \mathrm{Mpc}$ ) are marked by the shaded areas

The average HI mass of the galaxies in the Local Volume is $4.610^{7} M_{\odot}$.

The HI masses in Fig. 6 cover a range from $10^{6}$ to $10^{10}$ solar masses. The HI luminosity function for galaxies has been studied with galaxies of $10^{7}$ and more solar masses in HI so far. With the data of the new dwarf galaxies within the Local Volume we will be able in the end to discuss the HI luminosity function starting from $10^{6}$ solar masses.

The galaxies in our sample have small line widths on the average. In Fig. 7 we present the distribution of observed line widths in the upper panel and the (for inclination) corrected line widths in the lower panel. The optical axial ratio has been used here to derive the inclination. Galaxies within the Local Volume are indicated by the shaded areas. The peak of the line width distribution of the galaxies within the Local Volume is $39 \mathrm{~km} \mathrm{~s}^{-1}$ for the uncorrected and $47 \mathrm{~km} \mathrm{~s}^{-1}$ for the corrected line widths.

The three global parameters we have considered so far point altogether toward the dwarfish character of the Local Volume objects in our sample: the average linear diameter of $1.4 \pm 0.2 \mathrm{kpc}$ (Fig. 5), the mean total HI mass of $4.610^{7} M_{\odot}$ and the small line width of less than $50 \mathrm{~km} \mathrm{~s}^{-1}$.

Two more global parameters are shown in Fig. 8, pseudo HI surface density $\Sigma_{\mathrm{HI}}$ and the relative $\mathrm{HI}$ content $M_{\mathrm{HI}} / M_{\mathrm{T}}$. The pseudo HI surface density is obtained by dividing the total HI mass $M_{\mathrm{HI}}$ of the galaxy by the disk area of the galaxy as defined by its optical diameter $A_{0}$. This quantity is given in units of solar mass per square 


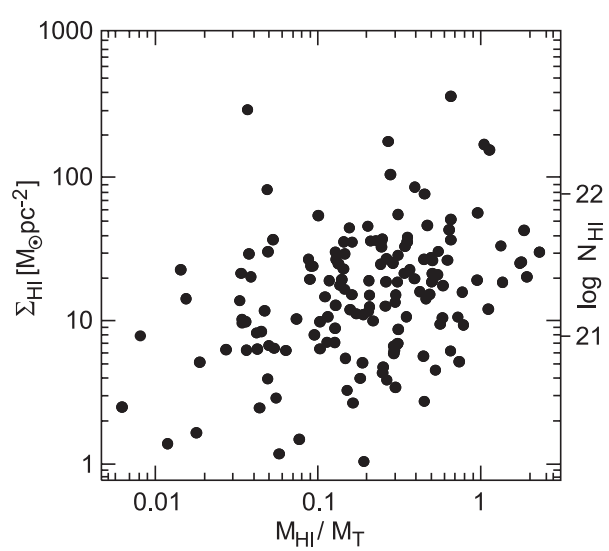

Fig. 8. The pseudo column density of neutral hydrogen $\left(\Sigma_{\mathrm{HI}}\right.$ in $\left.M_{\odot} \mathrm{pc}^{-2}\right)$ of our sample as plotted versus the relative HIcontent $\left(M_{\mathrm{HI}} / M_{\mathrm{T}}\right)$

parsec as well as in the usual HI column density $N_{\mathrm{HI}}$ in atoms $\mathrm{cm}^{-2}$. This quantity is plotted versus the relative HI content $M_{\mathrm{HI}} / M_{\mathrm{T}}$. Our galaxies fill the usual range in $\mathrm{HI}$ surface density as well as in relative HI content as observed for normal galaxies (e.g. HR). The present sample of galaxies is relatively rich in HI. Some of the scatter in the diagram is due to uncertainties in observed quantities, especially the inclination which is used to correct the line width which itself enters the total mass calculation by the square. The optical diameters are uncertain for galaxies at low galactic latitudes due to the high foreground extinction, e.g. Cas 2, ESO 137-G27, BK12, ESO 558-11. If we exclude the confused galaxies and those with heavy galactic extinction all entries in Fig. 8 with $\Sigma_{\mathrm{HI}} \geq$ $100 M_{\odot} \mathrm{pc}^{-2}$ are gone. Low values of the HI surface density are not only due to the uncertainties of observational data, the gas content of dwarf galaxies is very sensitive to outside influences (tidal interactions) due to their shallow gravitational potential.

Finally we plot the HI surface brightness versus the optical surface brightness (Fig. 9). The surface brightness class (Table 1, Col. 7) has been coded from 4 to 1 from high SB to extremely low SB in steps of 1 . The different errors of the mean values of each class essentially depend on the different population size of each SB class. However, there is a definite trend of the HI surface density to grow with increasing optical SB by a factor of 2 to 4 (e.g. van der Hulst et al. 1993; de Blok 1997).

\section{Conclusion}

In this paper we presented an HI search for 257 candidates for nearby dwarf galaxies. A detection rate of $60 \%$ on the average is quite high keeping in mind the limited velocity band and the fact that single-dish telescopes are literally "blind" for weak emission in the velocity range of

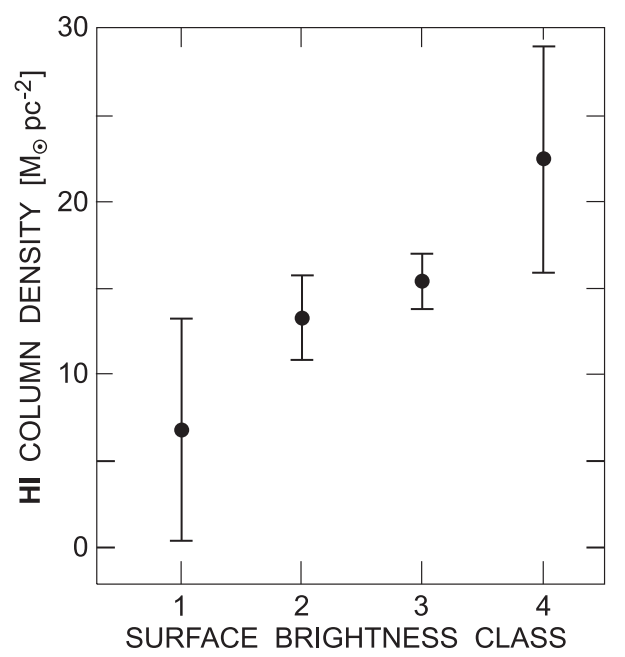

Fig. 9. This figure presents a correlation between the pseudo HI column density with the optical surface brightness of the galaxy in our actual sample. The surface brightness class is taken from KK98; 1 = extremely low, $2=$ very low, $3=$ low, $1=$ high SB. The error bars correspond to twice the rms error of the mean of each SB class

the local HI emission (i.e. within -140 to $+20 \mathrm{~km} \mathrm{~s}^{-1}$ ) and for $20 \%$ of HI-poor (spheroidal and Sph/Ir) objects in the sample. Most of the detected galaxies are located within the local supercluster, and about $25 \%$ are members of the Local Volume. The dwarfs within the Local Volume have a mean linear diameter of $1.4 \pm 0.2 \mathrm{kpc}$, a mean observed linewidths of $39 \mathrm{~km} \mathrm{~s}^{-1}$, and a mean total HI mass of 4.6 $10^{7} M_{\odot}$. The smallest galaxies have HI masses of just over $10^{6}$ solar masses. Once this full-sky survey will be finished we will be able to discuss the luminosity function of the Local Volume including these tiny dwarf galaxies. This investigation is especially needed as recent determinations of the galaxy luminosity function exhibit an increase for low mass objects. The exact value of this increase will be important for deriving the mass density in the local universe.

Acknowledgements. The Australia Telescope is funded by the Commonwealth of Australia for operation as a National Facility managed by CSIRO.

The Nançay Radio Astronomy Observatory is the Unité Scientifique de Nançay of the Observatoire de Paris, associated as Unité de Service et de Recherche (USR) No. B704 to the French Centre National de la Recherche Scientifique (CNRS). The Observatory also gratefully acknowledges the financial support of the Conseil Régional of the Région Centre in France.

This research has made use of the NASA/IPAC Extragalactic Database (NED) which is operated by the Jet Propulsion Laboratory, California Institute of Technology, under contract with the National Aeronautics and Space Administration. 
This work has been partially supported by the Deutsche Forschungsgemeinschaft (DFG) under project No. 436 RUS 113/470/0 and Eh 154/1-1.

\section{References}

Burton W.B., Verheijen E.B., Kraan-Korteweg R.C., Henning P.A., 1996, A\&A 293, L33

Cote S., Freeman K.C., Carignan C., Quinn P.J., 1997, AJ 114, 1313

de Blok W.J.G., 1997, Ph.D. Thesis, University of Groningen

Gallagher J.S., Littleton J.E., Mathews L.D., 1995, AJ 109, 2003

Hartmann D., Burton W.B., 1997, Atlas of Galactic Neutral Hydrogen. Cambridge University Press, Cambridge

Huchra J., 1995, A catalog of galaxy redshifts

Huchtmeier W.K., Bohnenstengel H.-D., 1981, A\&A 100, 72

Huchtmeier W.K., Lercher G., Seeberger R., Saurer W., Weinberger R., 1995, A\&A 293, L33

Huchtmeier W.K., van Driel W., 1996, A\&A 305, L25

Huchtmeier W.K., Karachentsev I.D., Karachentseva V.E., 1997, A\&A 322, 375
Huchtmeier W.K., Richter O.G., 1988, A\&A 203, 237 (HR)

Huchtmeier W.K., Richter O.-G., 1989, A general catalog of HI observations of galaxies. Springer-Verlag, New York van der Hulst J.M., Skillman E.D., Smith T.R., Bothun G.D., McGaugh S.S., de Blok W.J.G., 1993, AJ 106, 548

Karachentsev I.D., Makarov D.I., Huchtmeier W.K., 1999, A\&AS (in press)

Karachentseva V.E., Karachentsev I.D., 1998, A\&AS 127, 409

Karachentseva V.E., Karachentsev I.D., Richter G.M., 1999, A\&AS 135, 221

Kraan-Korteweg R.C., Tammann G.A., 1979, Astron. Nachr. 300, 181 (KKT)

Kraan-Korteweg R.C., Loan A.J., Burton W.B., et al., 1994, Nat 372,77

Matthews L.D., Gallagher J.S., Littleton J.E., 1995, AJ 110, 581

Paturel G., Fouque P., Bottinelli L., Gouguenheim L., 1992, Catalogue of Principal Galaxies, Lyon (PGC)

Roberts M.S., 1969, AJ 74, 859

Schombert J.M., Bothun G.D., Schneider S.E., McGaugh S.S., 1992, AJ 103, 1107

Tully R.B., 1988, Nearby Galaxy Catalog. Cambridge Univ. Press 
Table 1. List of new Local Volume dwarf candidates

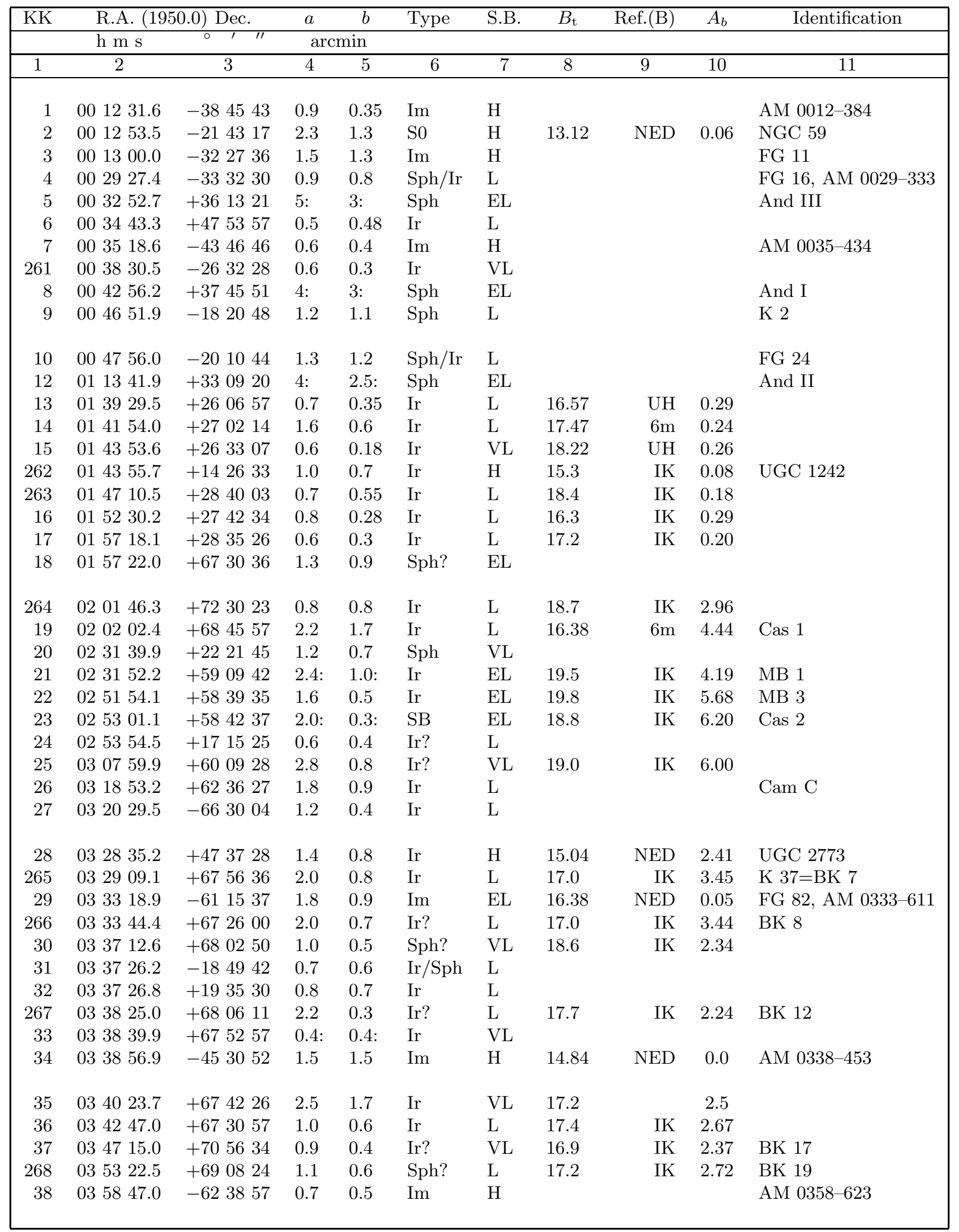


Table 1. continued

\begin{tabular}{|c|c|c|c|c|c|c|c|c|c|c|}
\hline KK & \multicolumn{2}{|c|}{ R.A. (1950.0) Dec. } & $a$ & $b$ & Type & S.B. & $B_{\mathrm{t}}$ & Ref.(B) & $A_{b}$ & Identification \\
\hline \multicolumn{3}{|c|}{$\mathrm{h} \mathrm{m} \mathrm{s}$} & \multicolumn{2}{|c|}{$\operatorname{arcmin}$} & & & & & & \\
\hline 1 & 2 & 3 & 4 & 5 & 6 & 7 & 8 & 9 & 10 & 11 \\
\hline 269 & 035934.8 & +712544 & 1.2 & 0.5 & Ir & VL & 17.0 & IK & 1.17 & BK 21 \\
\hline 39 & 040006.0 & +712000 & 1.1: & $0.9:$ & Ir? & EL & 18.5 & IK & 1.12 & \\
\hline 40 & 040556.0 & -552721 & 1.6 & 1.0 & $\operatorname{Im}$ & $\mathrm{H}$ & 14.73 & NED & 0.0 & AM $0405-552$ \\
\hline 270 & 040644.0 & +703833 & 0.9 & 0.4 & $\mathrm{Ir}$ & $\mathrm{L}$ & 16.6 & IK & 1.17 & \\
\hline 41 & 041926.7 & +724127 & 3.7 & 2.1 & Sph? & VL & & & & Cam A \\
\hline 42 & 043944.4 & +611547 & 0.6 & 0.6 & Ir? & VL & & & & \\
\hline 43 & 044511.0 & -360018 & 2.2 & 0.8 & $\mathrm{Im} / \mathrm{Sm}$ & $\mathrm{H}$ & 15.23 & NED & 0.0 & AM $0445-360$ \\
\hline 271 & 044640.8 & +670429 & 0.6 & 0.35 & Sph? & $\mathrm{L}$ & 17.7 & IK & 0.92 & \\
\hline 44 & 044803.3 & +670102 & 2.2 & 1.1 & Ir & $\mathrm{L}$ & 16.71 & UH & 0.93 & \\
\hline 45 & 052135.2 & -343713 & 0.55 & 0.4 & $\operatorname{Im}$ & $\mathrm{H}$ & 16.8 & IK & 0.0 & AM $0521-343$ \\
\hline 46 & 052305.4 & -870514 & 1.4 & 0.7 & $\operatorname{Im}$ & $\mathrm{L}$ & 15.90 & NED & 0.61 & FG 154, AM $0522-870$ \\
\hline 47 & 052749.0 & -873736 & 1.0 & 0.45 & $\operatorname{Im}$ & $\mathrm{L}$ & & & & AM $0528-873$ \\
\hline 48 & 052826.3 & -245444 & 1.7: & $0.3:$ & $\mathrm{Im} / \mathrm{Sm}$ & VL & 16.17 & NED & 0.06 & AM $0528-245$ \\
\hline 49 & 053900.7 & +063928 & 0.7 & 0.5 & $\operatorname{Im} ?$ & $\mathrm{H}$ & 16.1 & IK & 2.85 & \\
\hline 50 & 054725.5 & +025210 & 0.5 & 0.4 & Ir & VL & 18.3 & IK & 2.87 & \\
\hline 51 & 054847.9 & +025348 & 2.1 & 0.5 & $\mathrm{Ir}$ & EL & & & & \\
\hline 52 & 060218.4 & -193703 & 1.2 & 0.5 & Ir & $\mathrm{L}$ & 17.06 & NED & 0.38 & \\
\hline 53 & 061251.5 & -513141 & 1.1 & 0.6 & $\operatorname{Im}$ & $\mathrm{L}$ & 16.54 & NED & 0.15 & AM $0612-513$ \\
\hline 54 & 062416.7 & -261406 & 0.6 & 0.3 & Ir & $\mathrm{H}$ & 15.6 & IK & 0.43 & AM 0624-261 \\
\hline 55 & 063755.8 & -404024 & 0.7 & 0.45 & Ir & VL & 16.23 & NED & 0.33 & AM 0637-404 \\
\hline 56 & 063949.0 & +364103 & 1.3 & 0.4 & Ir? & $\mathrm{L}$ & 17.9 & IK & 0.66 & \\
\hline 57 & 070449.9 & -215729 & 1.9 & 1.1 & Ir & $\mathrm{L}$ & 15.8 & IK & 2.81 & \\
\hline 58 & 070756.2 & -512308 & 1.4 & 1.1 & $\mathrm{Ir}$ & $\mathrm{L}$ & 15.31 & NED & 0.25 & FG 203 \\
\hline 59 & 071741.2 & -571906 & 2.1 & 1.6 & $\mathrm{Im} / \mathrm{Sm}$ & VL & 16.1 & IK & 0.43 & FG 206, AM 0717-571 \\
\hline 60 & 072023.0 & +460610 & 1.1 & 0.4 & Ir & $\mathrm{L}$ & & & & \\
\hline 61 & 072913.1 & +665940 & 3: & $2:$ & Sph & VL & & & & DDO 44 \\
\hline 62 & 073150.6 & +421213 & 0.6 & 0.4 & Ir & $\mathrm{L}$ & 17.6 & IK & 0.21 & \\
\hline 64 & 073930.0 & +694109 & 0.6 & 0.2 & $\mathrm{Ir}$ & $\mathrm{L}$ & 16.6 & IK & 0.12 & \\
\hline 65 & 073940.2 & +164047 & 0.9 & 0.5 & $\operatorname{Ir}$ & $\mathrm{H}$ & 15.6 & NED & 0.09 & \\
\hline 66 & 074405.4 & +401842 & 0.7 & 0.4 & $\operatorname{Ir}$ & $\mathrm{L}$ & 17.0 & IK & 0.22 & \\
\hline 67 & 080034.9 & +151703 & 1.0 & 0.5 & Ir & $\mathrm{L}$ & & & & \\
\hline 68 & 082717.0 & -845857 & 1.1 & 1.0 & $\operatorname{Ir} ?$ & $\mathrm{H}$ & & & & \\
\hline 69 & 084944.1 & +335913 & 2.4: & 1.8: & Sph? & EL & 16.8 & IK & 0.07 & \\
\hline 70 & 085216.3 & +334502 & 1.1 & 1.0 & Sph? & EL & & & & \\
\hline 71 & 090656.7 & -230951 & 0.45 & 0.35 & $\mathrm{Ir} / \mathrm{Sph}$ & $\mathrm{L}$ & 18.7 & IK & 0.73 & AM 0906-231 \\
\hline 72 & 090928.5 & -234635 & 0.6 & 0.5 & $\mathrm{Sph}$ & $\mathrm{L}$ & & & & \\
\hline 73 & 091015.6 & -240205 & 0.9 & 0.8 & Sph & $\mathrm{L}$ & & & & \\
\hline 74 & 091218.0 & -232055 & 0.8 & 0.4 & $\operatorname{Im}$ & $\mathrm{L}$ & & & & FG 247, AM 0912-232 \\
\hline 75 & 091248.9 & -254030 & 0.9 & 0.6 & Ir & $\mathrm{L}$ & 18.0 & IK & 0.73 & \\
\hline U5005 & 092137.4 & +222920 & 1.3 & 0.9 & $\mathrm{~S} ?$ & $\mathrm{H}$ & 15.9 & IK & 0.10 & UGC 5005 \\
\hline 76 & 093823.6 & -762141 & 2.1 & 0.8 & Ir & $\mathrm{L}$ & & & & \\
\hline 77 & 094608.5 & +674425 & 2.4 & 1.8 & Sph & VL & & & & \\
\hline 78 & 094723.6 & +314126 & 0.5 & 0.3 & Ir & $\mathrm{H}$ & 17.6 & IK & 0.03 & \\
\hline 79 & 095003.5 & +293246 & 0.6 & 0.4 & BCD? & $\mathrm{H}$ & 17.0 & IK & 0.02 & \\
\hline 80 & 095045.0 & +294057 & 1.1 & 0.6 & Ir & $\mathrm{L}$ & & & & \\
\hline
\end{tabular}


Table 1. continued

\begin{tabular}{|c|c|c|c|c|c|c|c|c|c|c|}
\hline KK & \multicolumn{2}{|c|}{ R.A. (1950.0) Dec. } & $a$ & $b$ & Type & S.B. & $\overline{B_{\mathrm{t}}}$ & Ref.(B) & $\overline{A_{b}}$ & Identification \\
\hline & \multicolumn{2}{|l|}{$\mathrm{h} \mathrm{m} \mathrm{s}$} & \multicolumn{2}{|c|}{$\operatorname{arcmin}$} & & & & & & \\
\hline 1 & 2 & 3 & 4 & 5 & 6 & 7 & 8 & 9 & 10 & 11 \\
\hline 81 & 095300.8 & +684947 & 2.6 & 2.6 & Sph & VL & & & & K 61 \\
\hline 82 & 100025.3 & -055755 & 0.6 & 0.5 & Ir & $\mathrm{L}$ & 17.73 & NED & 0.05 & \\
\hline 82 & 100025.3 & -055755 & 0.6 & 0.5 & Ir & $\mathrm{L}$ & 17.73 & NED & 0.05 & \\
\hline 83 & 100118.0 & +664800 & 1.7 & 1.7 & Sph & VL & & & & DDO $71, \mathrm{~K} 63$ \\
\hline 84 & 100305.8 & -073020 & 1.9 & 1.3 & Sph & $\mathrm{L}$ & & & & K 65 \\
\hline 85 & 100309.0 & +680419 & 2.0 & 1.0 & Sph & $\mathrm{L}$ & & & & K 64, UGC 5442 \\
\hline 86 & 100522.0 & +304409 & 1.0 & 0.6 & $\operatorname{Ir} ?$ & $\mathrm{~L}$ & & & & MCG 5-24-18 \\
\hline 87 & 101237.6 & -443608 & 1.2 & 1.0 & $\mathrm{Sm} ?$ & $\mathrm{H}$ & & & & AM $1012-443$ \\
\hline 88 & 101357.4 & -394423 & 0.9 & 0.5 & Ir & VL & & & & AM 1013-394 \\
\hline 89 & 102247.6 & +675432 & 2.0 & 2.0 & Sph & VL & 15.8 & NED & 0.06 & DDO 78 \\
\hline 90 & 102626.1 & +230157 & 1.0 & 0.15 & $\mathrm{Ir} / \mathrm{S}$ & VL & 16.8 & IK & 0.0 & \\
\hline 91 & 103100.0 & +661600 & 1.0 & 0.8 & Sph & VL & & & & $\mathrm{BK} 6 \mathrm{~N}$ \\
\hline 92 & 103330.1 & +274752 & 0.8 & 0.7 & Ir & $\mathrm{L}$ & 16.9 & IK & 0.04 & \\
\hline 93 & 104345.8 & +141716 & 1.2 & 1.1 & Ir & VL & & & & \\
\hline 94 & 104418.1 & +131548 & 1.2 & 0.6 & Ir & VL & 17.9 & IK & 0.04 & \\
\hline 95 & 104603.6 & +645920 & $2.2:$ & 1.7: & Ir & VL & & & & UGCA 220 \\
\hline 96 & 104748.8 & +123734 & $1.2:$ & $0.8:$ & Sph & EL & & & & \\
\hline 97 & 105535.3 & +202235 & 0.7 & 0.5 & $\mathrm{Ir}$ & $\mathrm{L}$ & & & & \\
\hline 98 & 110937.7 & +170132 & 0.9 & 0.4 & $\mathrm{Sph} / \mathrm{Ir}$ & VL & 17.4 & IK & 0.0 & F 640-3 \\
\hline 99 & 111111.2 & -474602 & 0.35 & 0.25 & $\mathrm{Im}$ & $\mathrm{H}$ & & & & AM 1111-474 \\
\hline 100 & 111122.9 & +113610 & 1.2 & 0.5 & Ir & VL & & & & \\
\hline 101 & 111419.1 & -322239 & 0.7 & 0.4 & Sph? & $\mathrm{L}$ & & & & AM 1114-322 \\
\hline 102 & 112021.2 & +194458 & 0.6 & 0.4 & Ir? & $\mathrm{L}$ & 17.1 & IK & 0.0 & F $570-3$ \\
\hline 103 & 112103.8 & +193152 & 0.6 & 0.4 & Sph & VL & & & & \\
\hline 104 & 112614.5 & +183325 & 1.1 & 0.7 & Ir? & VL & 17.1 & IK & 0.0 & F $571-10$ \\
\hline 105 & 112640.7 & +462323 & 0.8 & 0.5 & Ir? & $\mathrm{L}$ & 16.6 & IK & 0.0 & \\
\hline 106 & 112708.7 & +524054 & 0.8 & 0.7 & Sph? & VL & 16.7 & IK & 0.0 & K 78 \\
\hline 107 & 113140.1 & +172614 & 0.7 & 0.4 & Ir & $\mathrm{L}$ & & & & \\
\hline 108 & 113723.0 & +464529 & 0.7 & 0.6 & Sph? & VL & 17.7 & IK & 0.01 & \\
\hline 109 & 114433.5 & +435659 & 0.6 & 0.4 & Ir? & $\mathrm{L}$ & 17.5 & IK & 0.0 & \\
\hline 110 & 114601.0 & +561144 & 0.6 & 0.6 & Ir? & $\mathrm{L}$ & & & & \\
\hline 111 & 115127.3 & +165955 & 0.6 & 0.45 & Ir? & $\mathrm{L}$ & 17.0 & IK & 0.14 & \\
\hline 113 & 115217.9 & +470459 & 0.5 & 0.3 & Ir? & VL & & & & \\
\hline 114 & 115355.1 & -362739 & 0.5 & 0.35 & $\mathrm{Im}$ & $\mathrm{H}$ & & & & \\
\hline 115 & 115537.1 & +490934 & 0.7 & 0.55 & $\mathrm{Im}$ & $\mathrm{H}$ & 16.2 & IK & 0.05 & MCG 8-22-48 \\
\hline 116 & 115618.5 & +460045 & 1.3 & 0.7 & Ir & $\mathrm{L}$ & 15.8 & IK & 0.0 & UGCA 259 \\
\hline 117 & 115724.6 & +445950 & 0.6 & 0.6 & Ir? & $\mathrm{L}$ & 17.1 & IK & 0.0 & \\
\hline 118 & 115842.9 & +540301 & 0.6 & 0.15 & Ir & VL & & & & \\
\hline 119 & 115913.5 & +283826 & 0.8 & 0.7 & Ir & $\mathrm{L}$ & 17.0 & IK & 0.0 & \\
\hline 120 & 120056.2 & -251135 & 2.5 & 1.9 & $\mathrm{Im} / \mathrm{Sm}$ & $\mathrm{L}$ & 16.8 & IK & 0.43 & FG 320;AM 1200-251 \\
\hline 121 & 120252.3 & +435913 & 1.1 & 0.6 & Ir & VL & & & & \\
\hline 122 & 120404.8 & +550238 & 1.0 & 0.25 & Ir & $\mathrm{L}$ & 16.4 & IK & 0.0 & \\
\hline 123 & 120420.5 & +173701 & 0.9 & 0.2 & Ir & $\mathrm{L}$ & & & & \\
\hline 124 & 120616.1 & +525029 & 0.5 & 0.3 & Ir? & EL & & & & \\
\hline 125 & 121016.9 & +691220 & 0.7 & 0.6 & Sph? & VL & & & & \\
\hline 126 & 121047.5 & +284144 & 0.6 & 0.3 & Ir & $\mathrm{L}$ & 16.7 & IK & 0.02 & \\
\hline
\end{tabular}


Table 1. continued

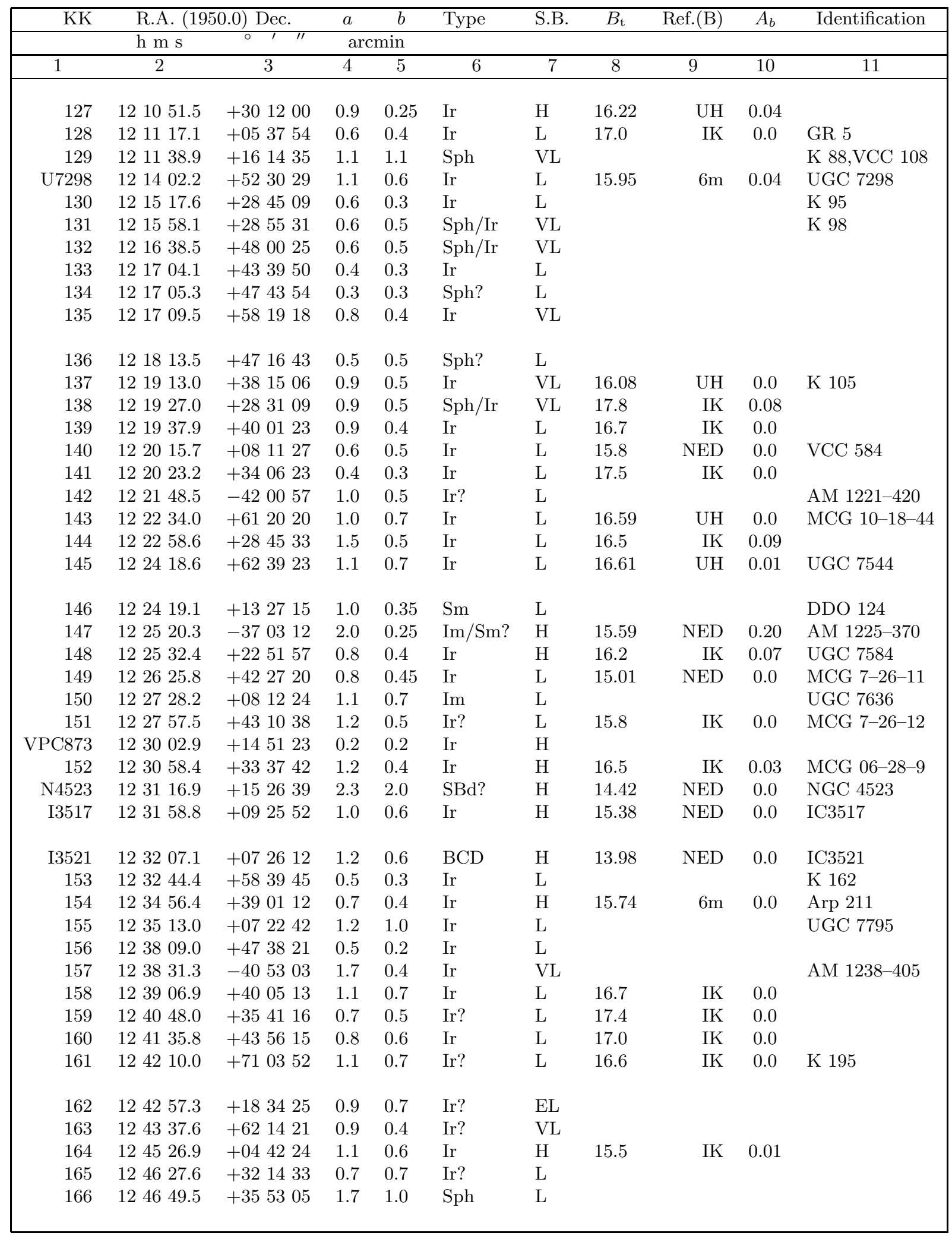


Table 1. continued

\begin{tabular}{|c|c|c|c|c|c|c|c|c|c|c|}
\hline KK & \multicolumn{2}{|c|}{ R.A. (1950.0) Dec. } & $a$ & $b$ & Type & S.B. & $B_{\mathrm{t}}$ & Ref.(B) & $A_{b}$ & Identification \\
\hline & \multicolumn{2}{|l|}{$\mathrm{h} \mathrm{m} \mathrm{s}$} & \multicolumn{2}{|c|}{$\operatorname{arcmin}$} & & & & & & \\
\hline 1 & 2 & 3 & 4 & 5 & 6 & 7 & 8 & 9 & 10 & 11 \\
\hline 167 & 124917.8 & +262256 & 0.8 & 0.5 & $\mathrm{Ir} ?$ & $\mathrm{~L}$ & 16.7 & IK & 0.02 & \\
\hline 168 & 125037.8 & +034244 & 0.5 & 0.4 & Ir? & VL & 17.3 & IK & 0.0 & \\
\hline 169 & 125041.3 & +125424 & 0.8 & 0.6 & $\mathrm{Ir}$ & $\mathrm{L}$ & 17.1 & IK & 0.05 & \\
\hline 170 & 125211.5 & -280412 & 0.8 & 0.6 & $\operatorname{Im}$ & $\mathrm{L}$ & 17.01 & NED & 0.30 & AM $1252-280$ \\
\hline 171 & 125303.1 & +331522 & 0.7 & 0.6 & Ir? & $\mathrm{L}$ & & & & \\
\hline 172 & 125414.0 & +121210 & 1.3 & 1.0 & Ir & $\mathrm{L}$ & 16.3 & IK & 0.07 & UGC 8061 \\
\hline 173 & 125607.5 & +180458 & 0.7 & 0.45 & Ir & $\mathrm{L}$ & 17.2 & & 0.11 & \\
\hline U8091 & 125610.5 & +142917 & 1.6 & 0.9 & $\mathrm{Ir}$ & $\mathrm{H}$ & 14.68 & NED & 0.04 & UGC 8091 \\
\hline 174 & 125629.7 & -492108 & 2.2 & 1.6 & Ir & $\mathrm{L}$ & 16.52 & NED & 0.89 & FG 363 \\
\hline 175 & 125638.7 & +354503 & 0.6 & 0.4 & $\operatorname{Ir} ?$ & $\mathrm{~L}$ & 17.1 & IK & 0.01 & \\
\hline 176 & 125717.1 & -190826 & 1.7 & 0.7 & $\mathrm{Sph} / \mathrm{Ir}$ & VL & 17.5 & IK & 0.17 & \\
\hline 177 & 130015.2 & +221602 & 0.8 & 0.7 & Sph? & VL & & & & F 575-1 \\
\hline 178 & 130032.8 & +262046 & 0.8 & 0.3 & Ir & $\mathrm{L}$ & & & & F 508-1 \\
\hline 180 & $\begin{array}{lll}13 & 02 & 02.1\end{array}$ & +180137 & 1.4 & 0.7 & Sph/Ir & VL & & & & F $575-4$ \\
\hline 181 & 130209.2 & +270231 & 0.7 & 0.6 & Ir & $\mathrm{L}$ & 17.25 & NED & 0.0 & F 508-v1 \\
\hline 182 & 130212.8 & -394854 & 1.0 & 0.55 & Ir & $\mathrm{L}$ & 16.33 & NED & 0.37 & \\
\hline 272 & 130333.1 & -493338 & 1.6 & 0.6 & $\mathrm{Ir}$ & $\mathrm{L}$ & & & & ESO 219-G027 \\
\hline 183 & 130414.8 & +181608 & 0.6 & 0.45 & Sph? & $\mathrm{L}$ & 17.9 & & 0.07 & \\
\hline 185 & 130636.8 & +332807 & 0.6 & 0.5 & Ir & $\mathrm{L}$ & & & & \\
\hline 186 & 130703.5 & -231635 & 0.7 & 0.3 & $\mathrm{Im}$ & $\mathrm{H}$ & & & & AM 1307-231 \\
\hline 187 & 130723.3 & -261943 & 1.0 & 0.8 & $\operatorname{Im}$ & $\mathrm{H}$ & 17.3 & IK & 0.31 & AM 1307-263 \\
\hline 188 & 130847.7 & +372639 & 0.9 & 0.6 & $\mathrm{Ir}$ & $\mathrm{L}$ & & & & \\
\hline 191 & 131124.8 & +421831 & 0.8 & 0.7 & Sph? & EL & 18.2 & IK & 0.0 & \\
\hline 192 & 131202.7 & +365008 & 0.7 & 0.5 & Sph? & $\mathrm{L}$ & 16.7 & IK & 0.0 & \\
\hline 193 & 131316.4 & +414555 & 0.6: & $0.6:$ & $\mathrm{Ir} / \mathrm{Sph}$ & EL & & & & \\
\hline 194 & 131507.3 & +443944 & 0.6 : & $0.4:$ & Ir? & VL & & & & \\
\hline 195 & 131820.5 & -311605 & 1.3 & 0.6 & $\mathrm{Ir}$ & VL & 17.4 & IK & 0.23 & \\
\hline 196 & 131849.9 & -444805 & 0.6 & 0.4 & $\mathrm{Ir} ?$ & $\mathrm{~L}$ & & & & AM 1318-444 \\
\hline 198 & 132007.0 & -331823 & 0.6 & 0.5 & Sph? & $\mathrm{L}$ & & & & \\
\hline 199 & 132031.4 & -285634 & 0.4 & 0.35 & $\mathrm{Ir}$ & EL & & & & \\
\hline 200 & 132148.1 & -304243 & 1.3 & 0.8 & $\mathrm{Im}$ & $\mathrm{H}$ & 16.67 & NED & 0.19 & K 15, AM 1321-304 \\
\hline 201 & 132220.0 & -372150 & 1.3 & 0.7 & $\mathrm{Ir} / \mathrm{Sph}$ & VL & & & & AM 1321-372 \\
\hline 202 & 132237.7 & -290039 & 0.6 & 0.45 & Ir & $\mathrm{L}$ & & & & \\
\hline 204 & 132528.7 & -375437 & 1.6 & 1.1 & Ir? & $\mathrm{L}$ & 14.97 & NED & 0.24 & FG 393, AM 1325-375 \\
\hline 205 & 132646.8 & +675328 & 1.2 & 0.5 & Ir & $\mathrm{L}$ & & & & UGC 8509 \\
\hline 206 & 133118.6 & +492130 & 1.0 & 0.6 & $\mathrm{Ir}$ & $\mathrm{H}$ & 14.6 & PGC & & MCG 8-25-18 \\
\hline 207 & 133131.6 & +564526 & 0.6 & 0.4 & Ir? & VL & & & & \\
\hline 208 & 133346.5 & -291900 & $6::$ & 2.5: & Ir & EL & 14.3 & & & \\
\hline 209 & 133552.8 & +492226 & 0.4 & 0.25 & $\mathrm{Ir}$ & $\mathrm{L}$ & & & & \\
\hline 210 & 133731.2 & -312647 & 1.4 & 0.6 & $\operatorname{Im} ?$ & $\mathrm{~L}$ & 16.57 & NED & 0.11 & FG 403 \\
\hline 212 & 133912.6 & +433231 & 0.8 & 0.6 & Ir? & $\mathrm{L}$ & 15.9 & IK & 0.0 & MCG 07-28-51 \\
\hline 215 & 134045.2 & -453922 & 0.5 & 0.5 & $\mathrm{Im}$ & $\mathrm{L}$ & & & & AM 1340-453 \\
\hline 216 & 134124.2 & +434243 & 1.1 & 0.5 & $\mathrm{Ir}$ & $\mathrm{H}$ & 14.8 & NED & 0.0 & UGC 8688 \\
\hline 218 & 134348.7 & -294347 & 1.7 & 0.7 & Sph? & VL & & & & \\
\hline 219 & 134521.9 & +393726 & 0.7 & 0.4 & $\operatorname{Ir} ?$ & $\mathrm{~L}$ & 17.3 & & & \\
\hline
\end{tabular}


Table 1. continued

\begin{tabular}{|c|c|c|c|c|c|c|c|c|c|c|}
\hline KK & \multicolumn{2}{|c|}{ R.A. (1950.0) Dec. } & $a$ & $b$ & Type & S.B. & $\overline{B_{\mathrm{t}}}$ & Ref.(B) & $\overline{A_{b}}$ & Identification \\
\hline & \multicolumn{2}{|l|}{$\mathrm{h} \mathrm{m} \mathrm{s}$} & \multicolumn{2}{|c|}{$\operatorname{arcmin}$} & & & & & & \\
\hline 1 & 2 & 3 & 4 & 5 & 6 & 7 & 8 & 9 & 10 & 11 \\
\hline 220 & 134522.2 & +332725 & $0.8:$ & $0.7:$ & Ir & $\mathrm{L}$ & 16.5 & IK & 0.0 & \\
\hline 223 & 134607.8 & +404808 & 0.4 & 0.3 & Ir? & $\mathrm{L}$ & 17.4 & IK & 0.0 & \\
\hline 224 & 134652.8 & +435054 & 1.0 & 0.4 & Ir & $\mathrm{L}$ & 16.9 & IK & 0.0 & \\
\hline 225 & 135251.5 & +375542 & 0.45 & 0.3 & Ir & $\mathrm{L}$ & & & & \\
\hline 226 & 135301.2 & -452447 & 1.1 & 0.7 & Ir & $\mathrm{L}$ & & & & \\
\hline 227 & 135403.7 & +403250 & 0.7 & 0.45 & Ir & $\mathrm{L}$ & & & & \\
\hline 228 & 135721.1 & +523616 & 0.9 & 0.8 & Ir? & $\mathrm{L}$ & 16.6 & IK & 0.0 & UGC 8914 \\
\hline 229 & 135954.3 & -465145 & 0.8 & 0.6 & Ir & $\mathrm{L}$ & 16.55 & NED & 0.49 & AM 1359-465 \\
\hline 230 & 140501.5 & +351809 & 0.6 & 0.5 & Ir & VL & 16.9 & IK & 0.0 & \\
\hline 274 & 140637.4 & -300230 & 0.8 & 0.6 & $\mathrm{Im}$ & $\mathrm{H}$ & 16.8 & IK & 0.22 & \\
\hline 231 & 141534.6 & +231821 & 0.8 & 0.3 & $\operatorname{Ir}$ & $\mathrm{L}$ & & & & \\
\hline 275 & 141600.0 & -450515 & 1.2 & 0.6 & $\operatorname{Im}$ & $\mathrm{H}$ & 14.62 & NED & 0.30 & AM $1415-450$ \\
\hline 232 & 144048.0 & +500140 & 0.9 & 0.5 & Ir & $\mathrm{VL}$ & 17.2 & IK & 0.05 & \\
\hline 233 & 144551.4 & +530231 & 1.1 & 0.7 & Ir & $\mathrm{L}$ & 15.9 & IK & 0.0 & MCG 9-24-40 \\
\hline 234 & 145713.6 & -513155 & 1.7 & 0.6 & Ir & $\mathrm{VL}$ & & & & FG 434 \\
\hline 236 & 150409.5 & +560324 & 0.9 & 0.7 & Sph? & VL & 19.17 & $\mathrm{UH}$ & 0.01 & \\
\hline 237 & 150646.1 & +562703 & 0.7 & 0.5 & Ir? & $\mathrm{L}$ & 18.21 & $\mathrm{UH}$ & 0.17 & K 233 \\
\hline 238 & 151159.0 & -225623 & 0.8 & 0.6 & $\operatorname{Im}$ & $\mathrm{L}$ & 17.11 & NED & 0.47 & FG 458, AM 1511-225 \\
\hline 239 & 152533.2 & -423636 & 1.9 & 0.7 & $\operatorname{Ir}$ & $\mathrm{L}$ & & & & FG 444 \\
\hline 240 & 162223.0 & -595033 & 2.9 & 0.7 & Sm? & $\mathrm{L}$ & 14.8 & IK & 0.90 & ESO 137- G27 \\
\hline 241 & 162259.4 & -602053 & 1.6 & 1.0 & Ir & VL & 16.6 & IK & 0.89 & FG 447 \\
\hline 242 & 175318.0 & +700841 & 0.8 & 0.6 & Sph? & EL & 19.0 & IK & 0.14 & \\
\hline 243 & 181805.0 & -621744 & 0.9 & 0.6 & Ir & $\mathrm{L}$ & 16.59 & NED & 0.44 & FG 458, AM 1818-622 \\
\hline 245 & 191617.0 & +635254 & 1.5 & 1.2 & $\mathrm{BCD}$ & $\mathrm{H}$ & & & & NGC 6789 \\
\hline 246 & 200048.0 & -314924 & $1.2:$ & $0.5:$ & Ir & $\mathrm{VL}$ & 17.06 & NED & 0.44 & FG 492 \\
\hline 247 & 200451.7 & -611230 & 0.9 & 0.6 & Ir & $\mathrm{L}$ & & & & AM 2004-611 \\
\hline 249 & 202558.8 & -315107 & 0.8 & 0.4 & Ir & $\mathrm{L}$ & 15.65 & NED & 0.26 & AM 2025-315 \\
\hline 250 & 202914.4 & +601622 & 1.8 & 0.8 & Ir & $\mathrm{VL}$ & 15.74 & $\mathrm{UH}$ & 1.31 & UGC 11583 \\
\hline 251 & 202931.9 & +601103 & 1.6 & 0.8 & $\operatorname{Ir} ?$ & $\mathrm{VL}$ & 16.49 & $\mathrm{UH}$ & 1.28 & \\
\hline 252 & 203033.5 & +603834 & 0.9 & 0.9 & Sph? & VL & 16.15 & $6 \mathrm{~m}$ & 1.95 & \\
\hline 253 & 203330.7 & -692158 & 1.0 & 0.9 & Ir & $\mathrm{L}$ & & & & AM 2033-692 \\
\hline 254 & 203346.2 & +605512 & 1.5 & 0.9 & $\operatorname{Ir} ?$ & EL & & & & \\
\hline 255 & 215421.4 & -603242 & 2.5 & 1.2 & Ir & $\mathrm{L}$ & 16.39 & NED & 0.0 & FG 532, AM 2154-603 \\
\hline 256 & 220904.6 & -432529 & 0.6 & 0.5 & $\mathrm{Im}$ & $\mathrm{L}$ & & & & AM 2209-432 \\
\hline 257 & 221925.2 & -483926 & 2.2 & 1.3 & Ir & $\mathrm{L}$ & 15.3 & IK & 0.0 & FG 545 \\
\hline 258 & 223756.3 & -310340 & 1.6 & 0.8 & $\operatorname{Ir} ?$ & $\mathrm{~L}$ & & & & K 20, FG 554, AM 2237-310 \\
\hline 259 & 230936.4 & -440301 & 4: & $2.2:$ & Sm? & $\mathrm{L}$ & & & & FG 569 \\
\hline 260 & 231146.9 & -435239 & 4.5 & 1.8 & $\operatorname{Ir}$ & VL & & & & \\
\hline
\end{tabular}


Table 2. List of new Local Volume dwarf candidates

\begin{tabular}{|c|c|c|c|c|c|c|c|c|c|}
\hline KK & HI-flux & $S_{\max }$ & velocity & line width & distance & $M_{B \mathrm{t}}$ & HI mass & $M_{\mathrm{HI}} / L_{\mathrm{B}}$ & Comments \\
\hline No. & $\mathrm{Jy} \mathrm{km} \mathrm{s}^{-1}$ & mJy & $\mathrm{kms}^{-1}$ & $\mathrm{~km} \mathrm{~s}^{-1}$ & $\mathrm{Mpc}$ & & $10^{7} M_{\odot}$ & & \\
\hline 1 & 2 & 3 & 4 & 5 & 6 & 7 & 8 & 9 & 10 \\
\hline 1 & & \pm 15 & & & & & & & ATCA \\
\hline 2 & 2.66 & $57 \pm 5.9$ & $361 \pm 2$ & 506276 & 5.4 & -15.70 & 1.9 & 0.06 & \\
\hline 3 & & \pm 24 & & & & & & & $\mathrm{~N}$ \\
\hline 4 & & \pm 35 & & & & & & & $\mathrm{~N}$ \\
\hline 5 & & \pm 3.5 & & & & & & & \\
\hline 6 & & \pm 4 & & & & & & & \\
\hline 7 & & \pm 20 & & & & & & & ATCA \\
\hline 261 & 2.6 & $87 \pm 13$ & $2694 \pm 2$ & 324041 & 35.9 & & & & \\
\hline 8 & & \pm 3.3 & & & & & & & \\
\hline 9 & & \pm 8 & & & & & & & \\
\hline 10 & & \pm 8.3 & & & & & & & \\
\hline 12 & & \pm 3.6 & & & & & & & \\
\hline 13 & 1.33 & $40 \pm 7$ & $357 \pm 9$ & 314552 & 7.0 & -13.15 & 1.5 & 0.53 & \\
\hline 14 & 3.04 & $74 \pm 6$ & $420 \pm 4$ & 365660 & 7.9 & -12.53 & 4.4 & 2.73 & \\
\hline 15 & 0.9 & $27 \pm 6$ & $368 \pm 4$ & 253740 & 7.2 & -11.69 & 1.1 & 1.51 & \\
\hline 262 & 1.1 & $18 \pm 7$ & $7356 \pm 3$ & 105124126 & 99.8 & -20.55 & 2.6 & 0.10 & \\
\hline 263 & 0.92 & $25 \pm 7$ & $3841 \pm 4$ & 384850 & 53.5 & -15.49 & 6.1 & 2.48 & \\
\hline 16 & 0.97 & $37 \pm 5$ & $206 \pm 3$ & 223234 & 4.9 & -12.76 & 5.6 & 0.28 & \\
\hline 17 & 0.95 & $28 \pm 5$ & $156 \pm 3$ & 345253 & 4.2 & -11.34 & 5.1 & 0.95 & \\
\hline 18 & & \pm 3.8 & & & & & & & \\
\hline 264 & 3.28 & $34 \pm 6$ & $2918 \pm 7$ & 128143155 & 42.0 & -17.38 & 130 & 0.98 & \\
\hline 19 & 46.4 & $930 \pm 4.6$ & $35 \pm 2$ & 49 & 3.5 & -15.87 & 14 & 0.39 & \\
\hline 20 & 11.18 & $760 \pm 2.2$ & $-70.2 \pm 1$ & 142022 & & & & & \\
\hline 21 & 9.0 & $160 \pm 10$ & $189 \pm 3$ & 607985 & 5.3 & -13.57 & 6.0 & 1.44 & \\
\hline 22 & 30.8 & $330 \pm 30$ & $96 \pm 2$ & $100116 .$. & 3.9 & -14.19 & & & \\
\hline 23 & 205 & $960 \pm 10$ & $112 \pm 2$ & 187202204 & 4.1 & -16.04 & 40.6 & 2.05 & \\
\hline 24 & & \pm 3.7 & & & & & & & \\
\hline 25 & 8.84 & $54 \pm 3.7$ & $2767 \pm 2$ & 260273277 & 39.5 & -19.35 & 330 & 0.38 & \\
\hline 26 & & \pm 2.6 & & & & & & & \\
\hline 27 & & \pm 6 & & & & & & & ATCA \\
\hline 28 & 26.1 & $349 \pm 30$ & $216 \pm 1$ & 819296 & 4.3 & -15.83 & 11.0 & 0.34 & \\
\hline 265 & 26.1 & $202 \pm 5$ & $1378 \pm 2$ & 144178187 & 21.1 & -18.33 & 280 & 0.83 & \\
\hline 29 & 0.95 & $33 \pm 3$ & $1152 \pm 5$ & 385256 & 12.6 & -14.38 & 3.6 & 0.41 & ATCA \\
\hline 266 & 54.24 & $629 \pm 5.4$ & $1434 \pm 1$ & 53119125 & 21.8 & -18.60 & 600 & 1.41 & \\
\hline 30 & 0.89 & $20 \pm 2.3$ & $1159 \pm 6$ & 455961 & 18.1 & -15.23 & 7.0 & 0.36 & \\
\hline 31 & & \pm 8.1 & & & & & & & \\
\hline 32 & & \pm 8 & & & & & & & \\
\hline $\begin{array}{r}267 \\
33\end{array}$ & 2.14 & $\begin{array}{c}32 \pm 3.1 \\
\quad \pm 5\end{array}$ & $1140 \pm 8$ & 99110 .. & 20.4 & -16.67 & 15.0 & 0.19 & \\
\hline 34 & 3.26 & $61 \pm 4$ & $1554 \pm 3$ & 607780 & 18.4 & -16.48 & 26.0 & 0.43 & ATCA \\
\hline 35 & 0.82 & $35 \pm 6$ & $119 \pm 3$ & $25 \ldots .$. & 2.1 & -12.11 & 0.085 & 0.08 & \\
\hline 36 & 3.45 & $33 \pm 2.8$ & $1267 \pm 7$ & 120134142 & 19.5 & -16.87 & 31.0 & 0.35 & \\
\hline 37 & 1.24 & $19.5 \pm 2.9$ & $836 \pm 5$ & 101109111 & 13.8 & -16.41 & 5.4 & 0.09 & \\
\hline 268 & 7.68 & $80 \pm 2.2$ & $1302 \pm 2$ & 120134138 & 20.0 & -17.20 & 71 & 0.61 & \\
\hline 38 & & \pm 7 & & & 7.3 & & & & ATCA \\
\hline \multicolumn{10}{|c|}{$\begin{array}{l}\mathrm{kk} 4 \text { : heliocentric velocity }=1651 \mathrm{~km} \mathrm{~s}^{-1}(\mathrm{NED}) \\
\mathrm{kk} 7,9,10 \text { : undetected in HI }(2) \\
\mathrm{kk} 8,12,5: \text { ANDI, II, III have been searched for HI within the radial velocity range }-550 \text { to } 770 \mathrm{~km} \mathrm{~s}^{-1} \\
\mathrm{kk} 18,26,32 \text { : undetected in HI (3) } \\
\mathrm{kk} 20 \text { : probably local HI } \\
\mathrm{kk} 35 \text { : resolved, companion to IC } 342\end{array}$} \\
\hline
\end{tabular}


Table 2. continued

\begin{tabular}{|c|c|c|c|c|c|c|c|c|c|}
\hline KK & HI-flux & $S_{\max }$ & velocity & line width & distance & $M_{B \mathrm{t}}$ & HI mass & $M_{\mathrm{HI}} / L_{\mathrm{B}}$ & Comment \\
\hline No. & $\mathrm{Jy} \mathrm{km} \mathrm{s}^{-1}$ & mJy & $\mathrm{km} \mathrm{s}^{-1}$ & $\mathrm{~km} \mathrm{~s}^{-1}$ & $\mathrm{Mpc}$ & & $10^{7} M_{\odot}$ & & \\
\hline 1 & 2 & 3 & 4 & 5 & 6 & 7 & 8 & 9 & 10 \\
\hline 269 & 6.83 & $69 \pm 5$ & $1734 \pm 2$ & 120133136 & 25.8 & -16.48 & 110 & 1.75 & \\
\hline 39 & 2.0 & $21 \pm 3.5$ & $1732 \pm 4$ & 123125126 & 25.7 & -14.73 & 17 & 1.42 & \\
\hline 40 & 2.87 & $53 \pm 5$ & $1066 \pm 2$ & 101109112 & 11.4 & -15.69 & 8.8 & 0.30 & ATCA \\
\hline 270 & 4.6 & $110 \pm 5$ & $1159 \pm 1$ & 425759 & 18.1 & -16.09 & 36 & 0.85 & \\
\hline 41 & & \pm 4.1 & & & & & & & \\
\hline 42 & & \pm 5 & & & & & & & \\
\hline 43 & 7.95 & $135 \pm 26$ & $1372 \pm 6$ & 46111130 & 15.7 & -16.04 & 46 & 1.14 & \\
\hline 271 & 0.79 & $12 \pm 4$ & $1581 \pm 25$ & & 23.4 & -15.22 & 10 & 0.54 & \\
\hline 44 & 4.47 & $220 \pm 15$ & $77 \pm 1$ & 182832 & 2.2 & -11.14 & 0.50 & 1.12 & \\
\hline 45 & 4.5 & $108 \pm 15$ & $955 \pm 3$ & 587883 & 9.9 & -13.26 & 10 & 3.29 & \\
\hline 46 & 1.34 & $54 \pm 5$ & $1745 \pm 3$ & 434850 & 21.0 & -16.69 & 14 & 0.19 & ATCA \\
\hline 47 & & \pm 6.4 & & & & & & & ATCA \\
\hline 48 & 6.87 & $72 \pm 4.9$ & $1850 \pm 2$ & 96120121 & 22 & -16.11 & 80 & 1.86 & \\
\hline 49 & 8.92 & $140 \pm 6.7$ & $455 \pm 2$ & 558487 & 4.8 & -15.26 & 4.9 & 0.25 & \\
\hline 50 & 2.08 & $21 \pm 5.1$ & $1776 \pm 4$ & 111117118 & 22.1 & -16.36 & 23 & 0.42 & \\
\hline 51 & & \pm 4.9 & & & & & & & \\
\hline 52 & 5.85 & $74 \pm 4.1$ & $883 \pm 2$ & 94105108 & 9.1 & -13.36 & 11 & 3.27 & \\
\hline 53 & 0.85 & $29 \pm 3$ & $1253 \pm 3$ & 344244 & 13.2 & -14.39 & 3.5 & 0.39 & ATCA \\
\hline 54 & 3.12 & $97 \pm 6.4$ & $491 \pm 2$ & 293539 & 3.4 & -12.71 & 0.89 & 0.47 & \\
\hline 55 & 1.26 & $53 \pm 5$ & $824 \pm 6$ & 516366 & 7.5 & -13.59 & 1.7 & 0.39 & ATCA \\
\hline 56 & 0.8 & $13 \pm 3$ & $2287 \pm 6$ & 588385 & 30.6 & -15.53 & 18.0 & 0.70 & \\
\hline 57 & 7.26 & $73 \pm 6$ & $738 \pm 3$ & 116132136 & 6.7 & -16.29 & 7.8 & 0.15 & \\
\hline 58 & 2.15 & $82 \pm 5$ & $1054 \pm 2$ & 273537 & 10.3 & -15.07 & 5.4 & 0.32 & ATCA \\
\hline 59 & 4.04 & $94 \pm 6$ & $1145 \pm 2$ & 214567 & 11.5 & -14.71 & 13.0 & 1.06 & ATCA \\
\hline 60 & & \pm 4.3 & & & & & & & \\
\hline 61 & & \pm 6 & & & & & & & \\
\hline 62 & 2.71 & $37 \pm 3.4$ & $3100 \pm 10$ & 94114124 & 41.5 & -15.90 & 110 & 3.07 & \\
\hline 64 & 2.90 & $25 \pm 3.5$ & $3867 \pm 5$ & 125153156 & 53.5 & -17.48 & 200 & 1.28 & \\
\hline 65 & 3.43 & $86 \pm 5$ & $279 \pm 5$ & 386068 & 2.2 & -11.39 & 0.39 & 0.70 & \\
\hline 66 & 1.61 & $17 \pm 4$ & $2976 \pm 9$ & 637376 & 39.7 & -16.38 & 60 & 1.08 & \\
\hline 67 & & \pm 4.7 & & & & & & & \\
\hline 68 & & \pm 6 & & & & & & & ATCA \\
\hline 69 & 3.58 & $154 \pm 6$ & $464 \pm 1$ & 203033 & 5.7 & -12.12 & 2.6 & 2.42 & \\
\hline 70 & & \pm 5 & & & & & & & \\
\hline 71 & 1.54 & $49 \pm 5.7$ & $179 \pm 3$ & 273841 & & & & & \\
\hline 72 & & \pm 6 & & & & & & & \\
\hline 73 & & \pm 5.3 & & & & & & & \\
\hline 74 & & \pm 5.2 & & & & & & & \\
\hline 75 & 1.40 & $27 \pm 5.1$ & $2866 \pm 4$ & 687981 & 34.6 & -15.54 & 40 & 1.56 & \\
\hline U5005 & 6.93 & $0.66 \pm 3.6$ & $3824 \pm 4$ & 109122126 & 49.7 & -17.79 & 390 & 1.92 & \\
\hline 76 & & \pm 7 & & & & & & & ATCA \\
\hline 77 & & \pm 5.5 & & & & & & & \\
\hline 78 & 15.8 & $204 \pm 4.9$ & $520 \pm 2$ & 8099104 & 6.3 & -11.56 & 15 & 22.92 & \\
\hline 79 & 1.2 & $20 \pm 4$ & $1638 \pm 8$ & 617390 & 21.0 & -14.75 & 13 & 1.01 & \\
\hline 80 & & \pm 3.3 & & & & & & & \\
\hline \multicolumn{10}{|c|}{ kk 42: undetected in HI (3) } \\
\hline \multicolumn{10}{|c|}{ kk 61: undetected in HI (6), companion of NGC 2403} \\
\hline \multicolumn{10}{|c|}{ kk 65: companion of UGC 3974} \\
\hline \multicolumn{10}{|c|}{$\mathrm{kk} 68: v=738 \mathrm{~km} \mathrm{~s}^{-1}(1)$} \\
\hline \multicolumn{10}{|c|}{ kk 69, 70: companion of NGC 2683} \\
\hline \multicolumn{10}{|c|}{ kk 71: local HI? } \\
\hline \multicolumn{10}{|c|}{ kk 74: undetected in HI (5) } \\
\hline $70, \mathrm{I}$ & Dיט-4 & & & & & & & & \\
\hline
\end{tabular}


Table 2. continued

\begin{tabular}{|c|c|c|c|c|c|c|c|c|c|}
\hline KK & HI-flux & $S_{\max }$ & velocity & line width & distance & $M_{B \mathrm{t}}$ & HI mass & $M_{\mathrm{HI}} / L_{\mathrm{B}}$ & Comment \\
\hline No. & $\mathrm{Jy} \mathrm{km} \mathrm{s}^{-1}$ & mJy & $\mathrm{kms}^{-1}$ & $\mathrm{~km} \mathrm{~s}^{-1}$ & $\mathrm{Mpc}$ & & $10^{8} M_{\odot}$ & $10^{9} M_{\odot}$ & \\
\hline 1 & 2 & 3 & 4 & 5 & 6 & 7 & 8 & 9 & 10 \\
\hline 81 & & \pm 8.5 & & & & & & & \\
\hline 82 & 3.94 & $165 \pm 6$ & $185 \pm 1$ & 213135 & & & & & \\
\hline 82 & 1.54 & $40 \pm 3.2$ & $3769 \pm 6$ & 354855 & 47.3 & -15.75 & 84 & 2.53 & \\
\hline 83 & & \pm 6.2 & & & & & & & \\
\hline 84 & & \pm 7 & & & & & & & \\
\hline 85 & & \pm 5.7 & & & & & & & \\
\hline 86 & & \pm 4.6 & & & & & & & \\
\hline 87 & & \pm 6 & & & & & & & ATCA \\
\hline 88 & & \pm 7 & & & & & & & ATCA \\
\hline 89 & 0.84 & $27 \pm 6.3$ & $2788 \pm 2$ & 326263 & 37.2 & -17.11 & 27 & 0.25 & \\
\hline 90 & 2.5 & $33 \pm 3.8$ & $1206 \pm 4$ & 95105108 & 14.9 & -14.66 & 13 & 1.15 & \\
\hline 91 & & \pm 6.8 & & & & & & & \\
\hline 92 & 2.74 & $46 \pm 3.9$ & $1371 \pm 3$ & 717981 & 17.5 & -14.39 & 19.0 & 2.19 & \\
\hline 93 & & \pm 6 & & & & & & & \\
\hline 94 & 1.52 & $42 \pm 3.5$ & $833 \pm 3$ & 285255 & 9.4 & -12.21 & 3.1 & 2.63 & \\
\hline 95 & & \pm 4.5 & & & & & & & \\
\hline 96 & & \pm 6.7 & & & & & & & \\
\hline 97 & & \pm 6.5 & & & & & & & \\
\hline 98 & 0.78 & $9 \pm 2.2$ & $1205 \pm 20$ & 48118120 & 14.7 & -13.68 & 4.0 & 0.87 & \\
\hline 99 & & \pm 6 & & & & & & & ATCA \\
\hline 100 & & \pm 6 & & & & & & & \\
\hline 101 & & \pm 26 & & & & & & & $\mathrm{~N}$ \\
\hline 102 & 1.58 & $19.6 \pm 3.3$ & $3366 \pm 5$ & 86117120 & 43.8 & -16.22 & 71 & 1.49 & \\
\hline 103 & & \pm 8.8 & & & & & & & \\
\hline 104 & 1.36 & $21 \pm 5.6$ & $1303 \pm 3$ & 62114116 & 16.2 & -14.08 & 8.4 & 1.26 & \\
\hline 105 & 2.04 & $28 \pm 3.7$ & $1555 \pm 3$ & 809698 & 21.4 & -15.18 & 21 & 1.16 & \\
\hline 106 & 0.28 & $15 \pm 4.2$ & $592 \pm 5$ & 233032 & 8.9 & -13.09 & 0.53 & 0.20 & \\
\hline 107 & & \pm 5 & & & & & & & \\
\hline 108 & 6.99 & $72 \pm 3.8$ & $736 \pm 3$ & 88110138 & 10.5 & -12.46 & 18 & 12.12 & \\
\hline 109 & 0.7 & $37 \pm 4.5$ & $214 \pm 3$ & 112336 & 3.4 & -10.28 & 0.19 & 0.95 & \\
\hline 110 & & \pm 4 & & & & & & & \\
\hline 111 & 1.79 & $34 \pm 3.8$ & $980 \pm 4$ & 415254 & 12.0 & -13.62 & 0.61 & 1.40 & \\
\hline 113 & & \pm 4.3 & & & & & & & \\
\hline 114 & & \pm 31 & & & & & & & $\mathrm{~N}$ \\
\hline 115 & 2.29 & $37 \pm 3$ & $826 \pm 4$ & 749194 & 12.0 & -14.32 & 7.4 & 0.90 & \\
\hline 116 & 5.81 & $129 \pm 3.1$ & $1151 \pm 2$ & 415254 & 16.1 & -15.41 & 35 & 1.53 & \\
\hline 117 & 0.44 & $14 \pm 3$ & $1171 \pm 10$ & 385054 & 16.3 & -13.96 & 2.5 & 0.42 & \\
\hline 118 & & \pm 4.8 & & & & & & & \\
\hline 119 & 0.71 & $23 \pm 3$ & $841 \pm 3$ & 344244 & 10.9 & -13.23 & 2.0 & 0.65 & \\
\hline 120 & 17.12 & $253 \pm 6.5$ & $1785 \pm 1$ & 708589 & 20.8 & -15.30 & 180 & 8.73 & \\
\hline 121 & & \pm 3.4 & & & & & & & \\
\hline 122 & 2.32 & $33 \pm 4$ & $850 \pm 2$ & 729193 & 12.7 & -14.52 & 8.5 & 0.85 & \\
\hline 123 & & \pm 7.6 & & & & & & & \\
\hline 124 & & \pm 4.6 & & & & & & & \\
\hline 125 & & \pm 4 & & & & & & & \\
\hline 126 & 1.24 & $25 \pm 3.5$ & $1028 \pm 10$ & 365762 & 13.5 & -14.17 & 5.1 & 0.71 & \\
\hline $\begin{array}{l}\mathrm{kk} 8 \\
\mathrm{kk} 8 \\
\mathrm{kk} 8 \\
\mathrm{kk} 8 \\
\mathrm{kk} 8 \\
\mathrm{kk} 9 \\
\mathrm{kk} 1 \\
\mathrm{kk} 1\end{array}$ & $\begin{array}{l}15^{\prime} \text { from } \mathrm{P} \\
\text { companior } \\
\text { heliocentri } \\
\text { heliocentri } \\
\text { highly pro } \\
\text { 96: near L } \\
\text { 3: heliocent } \\
\text { 8: NGC } 378\end{array}$ & $\begin{array}{l}\text { IC } 29086(v \\
\text { of NGC } 3115 \\
\text { velocity } 969 \\
\text { velocity } 263 \\
\text { ably membe } \\
\text { triplet } \\
\text { c velocity } 18 \\
(v=739 \mathrm{kn}\end{array}$ & $\begin{array}{l}=662 \mathrm{~km} \mathrm{~s}^{-1} \\
\mathrm{~km} \mathrm{~s}^{-1}(\mathrm{NE} \\
\text { and } 2982 \mathrm{kn} \\
\text { of } \mathrm{M} 81 \text { gro } \\
4 \mathrm{~km} \mathrm{~s}^{-1}(\mathrm{~N} \\
\left.\mathrm{s}^{-1}\right) \text { at } 7.6^{\prime}\end{array}$ & $\begin{array}{l}\mathrm{s}^{-1}(6) \\
\text { ip, HI emissic } \\
\text { ED) }\end{array}$ & probabl & not from & s object & & \\
\hline
\end{tabular}


Table 2. continued

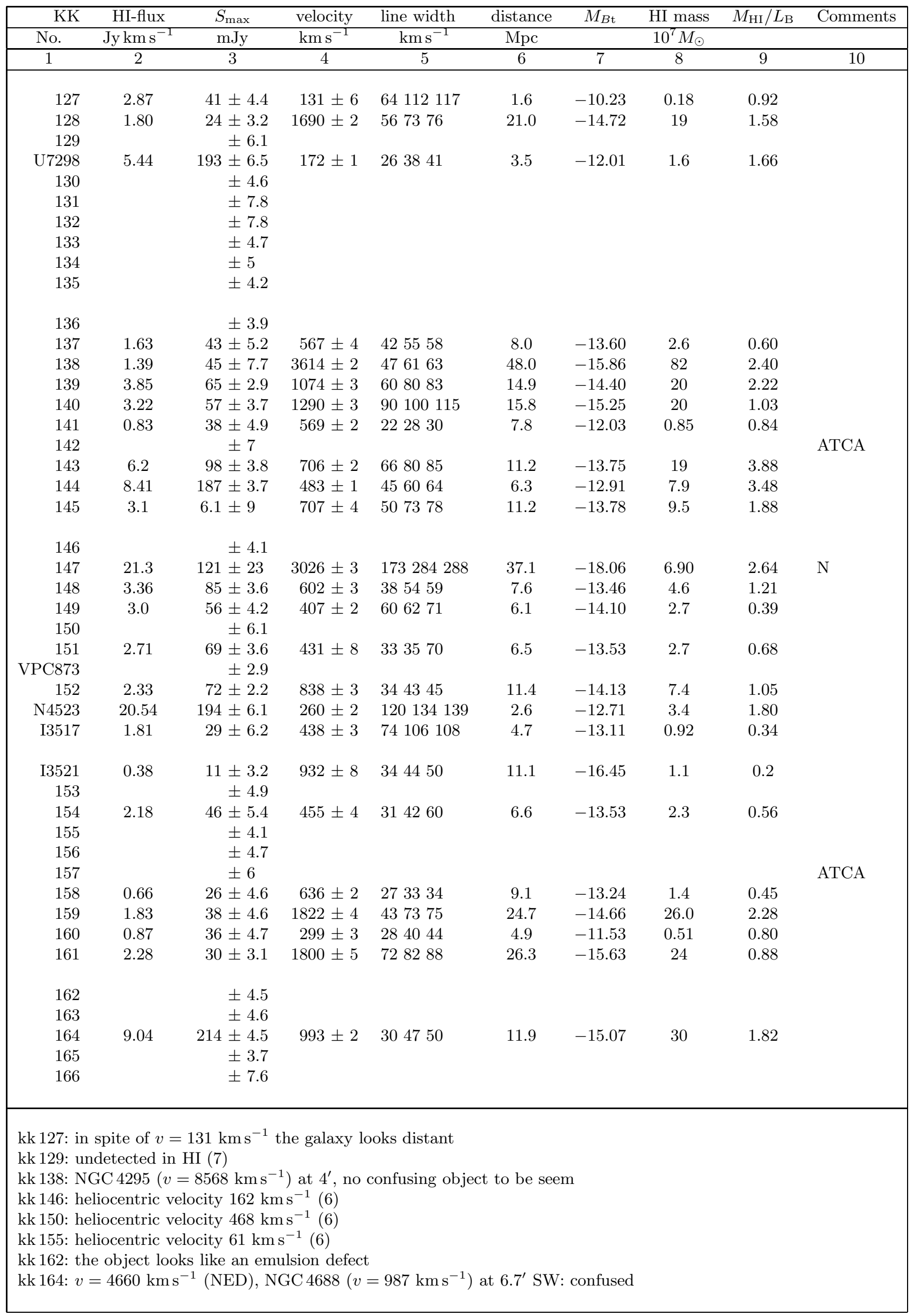


Table 2. continued

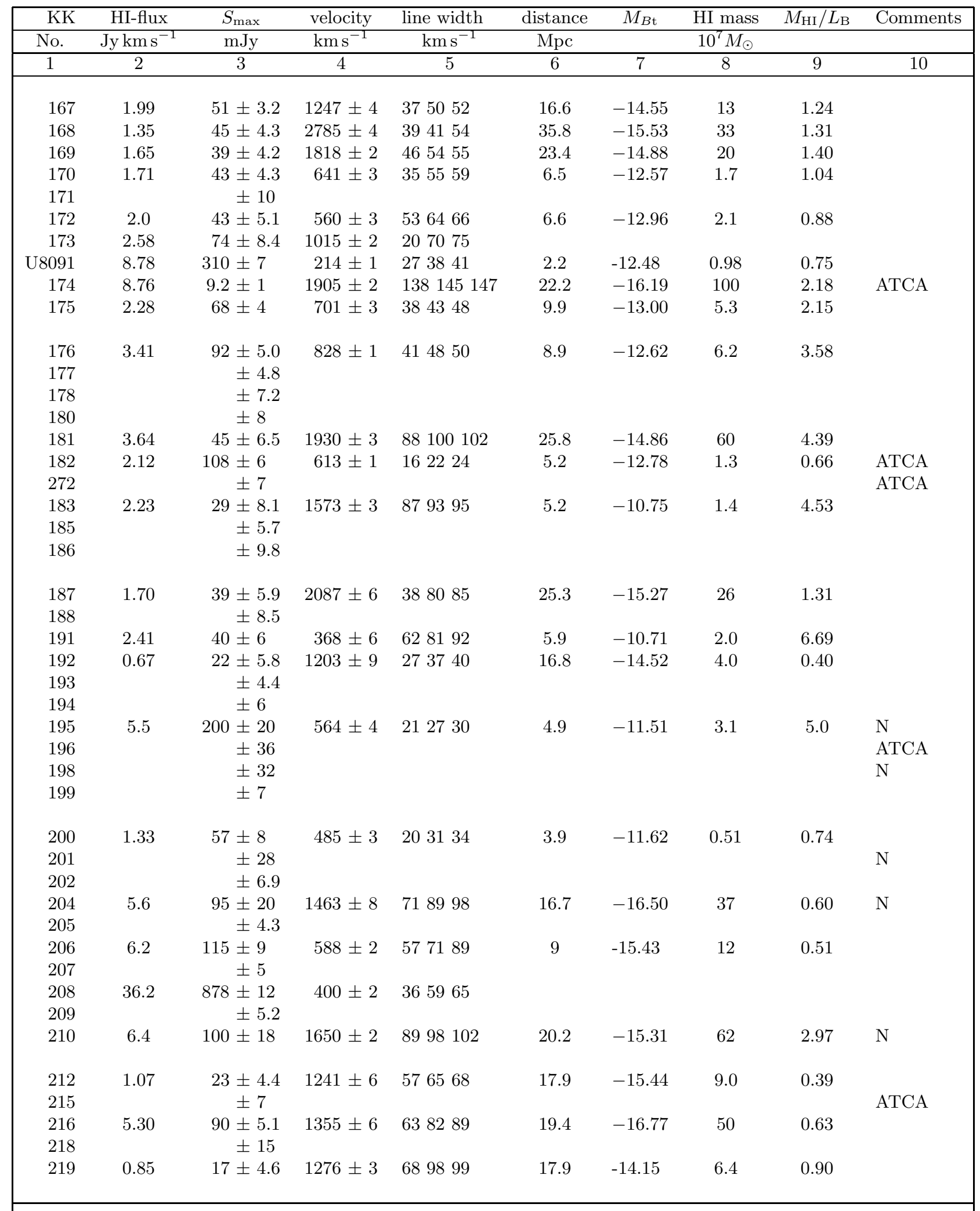

kk 170: HI detection by Matthewson \& Gallagher (1995)

kk 174: $v=1905 \mathrm{~km} \mathrm{~s}^{-1}$ conflicts with the galaxy morphology

$\mathrm{kk} 177,180$ : undetected in HI (7)

kk 191: NGC $5055\left(v=510 \mathrm{~km} \mathrm{~s}^{-1}, W=406 \mathrm{~km} \mathrm{~s}^{-1}\right)$ at $24.1^{\prime} \mathrm{E}$, possible confusion through far sidelobe

kk 192: NGC $5033\left(v=876 \mathrm{~km} \mathrm{~s}^{-1}, W=452 \mathrm{~km} \mathrm{~s}^{-1}\right)$ at $10.8^{\prime} \mathrm{W}$, different velocity range, no confusion

$\mathrm{kk} 205$ : undetected in $\mathrm{HI}(6)$

kk 208: $20^{\prime}$ from NGC 5236, confusion with the extended HI-halo of M 83 (4) 
Table 2. continued

\begin{tabular}{|c|c|c|c|c|c|c|c|c|c|}
\hline KK & HI-flux & $S_{\max }$ & velocity & line width & distance & $M_{B \mathrm{t}}$ & HI mass & $M_{\mathrm{HI}} / L_{\mathrm{B}}$ & Comments \\
\hline No. & $\mathrm{Jy} \mathrm{km} \mathrm{s}^{-1}$ & mJy & $\mathrm{km} \mathrm{s}^{-1}$ & $\mathrm{~km} \mathrm{~s}^{-1}$ & $\mathrm{Mpc}$ & & $10^{7} M_{\odot}$ & & \\
\hline 1 & 2 & 3 & 4 & 5 & 6 & 7 & 8 & 9 & 10 \\
\hline 220 & 0.8 & $33 \pm 5.6$ & $769 \pm 2$ & 273334 & 11.1 & -13.76 & 2.3 & 0.47 & \\
\hline 223 & 4.26 & $53 \pm 5.5$ & $2594 \pm 8$ & $\begin{array}{lll}69 & 130138\end{array}$ & 35.8 & -15.45 & 130 & 5.51 & \\
\hline 224 & 0.84 & $25 \pm 6.7$ & $1156 \pm 6$ & 264354 & 16.8 & -14.49 & 5.3 & 0.55 & \\
\hline 225 & & \pm 6.3 & & & & & & & \\
\hline 226 & & \pm 7 & & & & & & & ATCA \\
\hline 227 & & \pm 7.8 & & & & & & & \\
\hline 228 & 1.55 & $41 \pm 5.9$ & $1972 \pm 3$ & 446466 & 28.1 & -15.68 & 30 & 1.02 & \\
\hline 229 & 0.31 & $25 \pm 3$ & $1355 \pm 1$ & 142124 & 15.3 & -14.95 & 1.7 & 0.12 & ATCA \\
\hline 230 & 1.87 & $113 \pm 6.4$ & $61 \pm 1$ & 202932 & 1.9 & -9.57 & 0.17 & 1.58 & \\
\hline 274 & 6.3 & $57 \pm 15$ & $2598 \pm 6$ & 627378 & 32.5 & -16.06 & 160 & 3.79 & $\mathrm{~N}$ \\
\hline 231 & & \pm 7.1 & & & & & & & \\
\hline 275 & 9.08 & $106 \pm 5$ & $1633 \pm 3$ & 122148153 & 19.2 & -17.30 & 79 & 0.61 & ATCA \\
\hline 232 & 0.86 & $24 \pm 5.5$ & $2960 \pm 9$ & 374751 & 41.5 & -16.11 & 36 & 0.83 & \\
\hline 233 & 4.18 & $55 \pm 4.5$ & $724 \pm 2$ & 769193 & 11.9 & -14.60 & 14 & 1.26 & \\
\hline 234 & & \pm 6 & & & & & & & ATCA \\
\hline 236 & 5.18 & $20 \pm 6$ & $-150 \pm 1$ & 213436 & & & & & \\
\hline 237 & 5.22 & $174 \pm 5.6$ & $-177 \pm 1$ & 243437 & & & & & \\
\hline 238 & 2.03 & $32 \pm 5.9$ & $2435 \pm 9$ & 588892 & 36.0 & -16.22 & 64 & 1.34 & \\
\hline 239 & & \pm 6 & & & & & & & ATCA \\
\hline 240 & 5.2 & $30 \pm 3$ & $1371 \pm 5$ & 176188191 & 16.1 & -17.55 & 13 & 0.08 & ATCA \\
\hline 241 & 12.32 & $174 \pm 8$ & $1152 \pm 3$ & 659084 & 13.2 & -15.03 & 51 & 3.17 & ATCA \\
\hline 242 & 2.03 & $44 \pm 3$ & $426 \pm 6$ & 100118133 & 9.1 & -11.01 & 8 & 20.15 & \\
\hline 243 & 2.17 & & $957 \pm 2$ & 326070 & 11.0 & -14.18 & 6.8 & 0.78 & \\
\hline 245 & & \pm 4 & & & & & & & \\
\hline 246 & 8.3 & $149 \pm 30$ & $358 \pm 3$ & 536071 & 5.4 & -12.28 & 5.3 & 3.68 & $\mathrm{~N}$ \\
\hline 247 & & \pm 7 & & & & & & & ATCA \\
\hline 249 & 18.8 & $68 \pm 20$ & $2089 \pm 10$ & 124131158 & 28.5 & -17.09 & 360 & 2.93 & $\mathrm{~N}$ \\
\hline 250 & 20.0 & $244 \pm 6$ & $127 \pm 2$ & 90104107 & 5.6 & -14.54 & 15 & 1.44 & \\
\hline 251 & 14.62 & $223 \pm 8$ & $126 \pm 2$ & 648794 & 5.6 & -13.72 & 11 & 2.23 & \\
\hline 252 & 1.36 & $45 \pm 5$ & $132 \pm 3$ & 274750 & 5.6 & -14.56 & 1.1 & 0.10 & \\
\hline 253 & & \pm 7 & & & & & & & ATCA \\
\hline 254 & & \pm 8 & & & & & & & \\
\hline 255 & 1.16 & $63 \pm 4$ & $1682 \pm 1$ & 364448 & 21.1 & -15.45 & 12 & 0.52 & ATCA \\
\hline 256 & & \pm 7 & & & & & & & ATCA \\
\hline 257 & 3.36 & $70 \pm 5$ & $705 \pm 1$ & 778084 & 8.8 & -14.59 & 6.2 & 0.58 & ATCA \\
\hline 258 & & \pm 32 & & & & & & & \\
\hline 259 & & \pm 7 & & & & & & & ATCA \\
\hline 260 & & \pm 6 & & & & & & & ATCA \\
\hline \multicolumn{10}{|c|}{$\begin{array}{l}\text { kk 223: } 6.5 \text { arcmin NE of UGC } 8726\left(v=2334 \mathrm{~km} \mathrm{~s}^{-1}\right) \text {, confused } \\
\text { kk 227: companion of NGC } 5371 \\
\text { kk 236, 237: probably local HI } \\
\text { kk 242: near NGC 6503, no obvious confusing object within the antenna beam and 1st sidelobes } \\
\text { kk 245: resolved into stars with the } 6 \text {-m telescope (SAO), } v=-157 \mathrm{~km} \mathrm{~s}^{-1}(1) \\
\text { kk 254: undetected in HI (3) } \\
\text { kk 260: near NGC } 7531\end{array}$} \\
\hline \multicolumn{10}{|c|}{$\begin{array}{l}\text { 1. Huchra J. (1995) } \\
\text { 2. Gallagher J.S. et al. (1995). } \\
\text { 3. Huchtmeier W.K. et al. (1997). } \\
\text { 4. Huchtmeier W.K. et al. (1981). } \\
\text { 5. Matthews L.D. et al. (1995). } \\
\text { 6. Huchtmeier W.K. \& Richter O.G. (1989b). } \\
\text { 7. Schombert J.M. et al. (1992). }\end{array}$} \\
\hline
\end{tabular}

